\title{
Vortex beam: generation and detection of orbital angular momentum [Invited]
}

\author{
Yihua Bai (白毅华), Haoran Lv (吕浩然), Xin Fu (付 金金), and Yuanjie Yang (杨元杰) \\ School of Physics, University of Electronic Science and Technology of China, Chengdu 611731, China \\ *Corresponding author: dr.yang2003@uestc.edu.cn \\ Received August 29, 2021 | Accepted September 29, 2021 | Posted Online November 3, 2021
}

Recent advances in the research of vortex beams, structured beams carrying orbital angular momentum (OAM], have revolutionized the applications of light beams, such as advanced optical manipulations, high-capacity optical communications, and super-resolution imaging. Undoubtedly, the methods for generation of a vortex beam and detection of its OAM are of vital importance for the applications of vortex beams. In this review, we first introduce the fundamental concepts of vortex beams briefly and then summarize approaches to generating and detecting the vortex beams separately, from bulky diffractive elements to planar elements. Finally, we make a concise conclusion and outline that is yet to be explored.

Keywords: vortex beams; orbital angular momentum; topological charge.

DOI: 10.3788/COL202220.012601

\section{Introduction}

Light beams, as electromagnetic waves, can carry both energy and momentum. Meanwhile, we know that momentum can be classified into linear and angular momentum, and there are two particular kinds of angular momenta: spin angular momentum (SAM) and orbital angular momentum (OAM). More than a century ago, Poynting proposed that the SAM is related to the photon spin, namely, the circularly polarized light carries an SAM of $\pm \hbar$ per photon ${ }^{[1]}$, whereas linearly polarized light carries no SAM, as illustrated in Fig. 1(a). In 1932, Darwin realized the photon can carry both SAM and OAM, and he even obtained the expression for each one ${ }^{[2]}$. But, it was not until 1992 that Allen et al. revealed the OAM of a light beam clearly ${ }^{[3]}$. It was shown that vortex beams with an azimuthal phase factor $\exp (i l \theta)$ can carry OAM of $l \hbar$ per photon, where $l$ can be any integer number, named topological charge. The discovery of OAM of light has changed the way in which we understand and employ light. Different from traditional Gaussian beams, vortex beams exhibit a phase singularity in the center, which leads a doughnut-shaped intensity profile, as shown in Fig. 1(b). Figure 1(b) shows vortex beams with helical wavefronts, in which the number of intertwined helices and the handedness are dependent on the topological charge $l$.

Thus far, the most common mode for vortex beams is the Laguerre-Gaussian (LG) mode, because that mode is a solution of the Helmholtz equation in the cylindrical coordinate system under paraxial approximation ${ }^{[4]}$ :

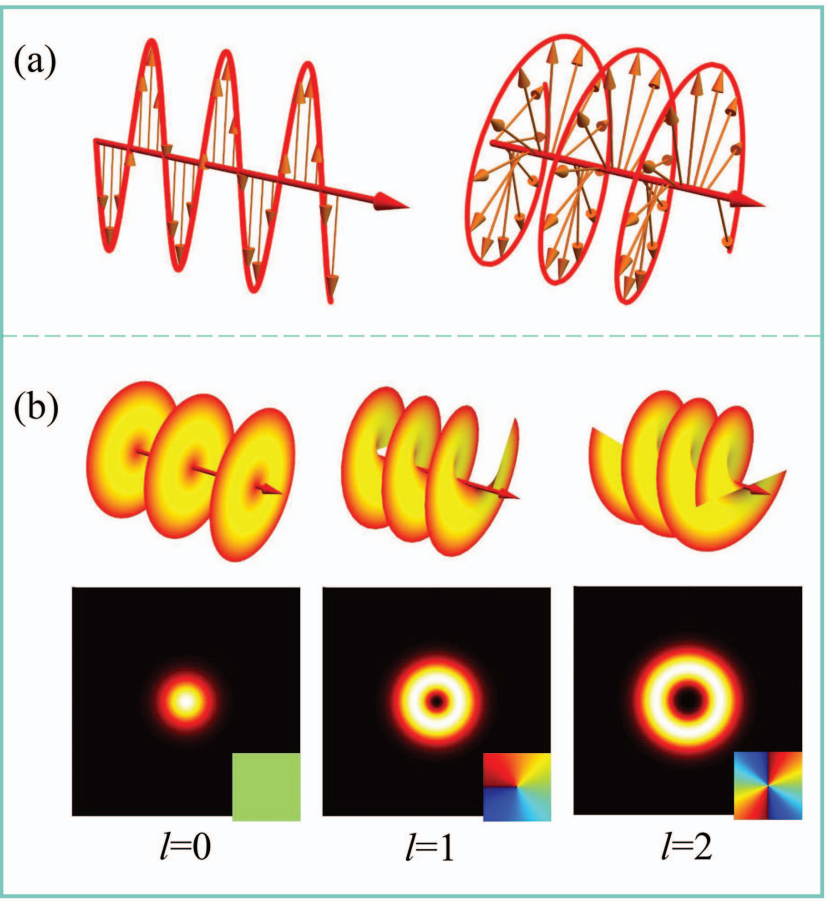

Fig. 1. (a) Linearly polarized light carries no SAM (left) and circularly polarized light carries an SAM of $\pm \hbar$ per photon (right). (b) Wavefront, intensity, and corresponding phase distribution of vortex beams with topological charge of $I=0,1,2$. 


$$
\begin{aligned}
\mathrm{LG}_{p l}(r, \phi, z) & =\sqrt{\frac{2 p !}{\pi(p+|l|) !}} \frac{1}{\omega(z)}\left[\frac{r \sqrt{2}}{\omega(z)}\right]^{|l|} \exp \left[\frac{-r^{2}}{\omega^{2}(z)}\right] L_{p}^{|l|}\left[\frac{2 r^{2}}{\omega(z)^{2}}\right] \\
& \times \exp \left[-\frac{r^{2}}{\omega(z)^{2}}\right] \exp (i l \phi) \\
& \times \exp \left[\frac{i k_{0} r^{2} z}{2\left(z^{2}+z_{R}^{2}\right)}\right] \exp \left[-i \eta \arctan \left(\frac{z}{z_{R}}\right)\right]
\end{aligned}
$$

where $\eta=2 p+|l|+1, z_{R}$ is the Rayleigh range, $L_{p}^{|l|}(x)$ is the associated Laguerre polynomial with $p$ being the number of radial nodes, and $\omega(z)=\omega(0)\left[\left(z^{2}+z_{R}^{2}\right) / z_{R}^{2}\right]^{1 / 2}$ denotes the $1 / e$ radius of the beam with $\omega(0)$ being the beam waist. Besides, there are other types of vortex beams such as non-zeroth-order Bessel beams ${ }^{[5]}$ and Mathieu beams ${ }^{[6]}$. Recent decades have witnessed several ground-breaking demonstrations of vortex beam in many aspects, ranging from optical communications ${ }^{[7]}$ and optical micro-manipulation ${ }^{[8]}$ to quantum information $^{[9]}$, etc.

In this review, a brief introduction about vortex beams is presented in Section 1, and approaches for generation and detection of OAM will be presented hereinafter. In Section 2, generation methods including classical optical elements, digital devices, photon sieve, metasurfaces, and other methods are summarized. In Section 3, detection methods by the use of interference, diffraction, geometric coordinate transformation, deep learning, and surface plasmon polariton are reviewed. Finally, we conclude and make a prospect in Section 4.

\section{Generation of Vortex Beams}

Hitherto, to enable emerging applications, varieties of techniques have been proposed for the generation of vortex beams over the past few decades ${ }^{[10,11]}$. Here, we review the generation methods including classical optical elements [such as mode conversion, spiral phase plate (SPP), and computer-generated holograms], digital devices [such as spatial light modulator (SLM) and digital micro-mirror device (DMD)], photon sieve, more recently metasurfaces, and other methods (such as q-plate and active vortex laser generators).

\subsection{Using classical optical elements}

\subsubsection{Mode conversion}

In the early years, laser beams carrying OAM were quickly developed both theoretically and experimentally. Particularly, Beijersbergen et al. first generated such beams experimentally by the use of two cylindrical lenses called a mode converter ${ }^{[12]}$, where LG modes of arbitrary order can be transformed directly from the Hermit-Gaussian (HG) modes by introducing a Gouy phase shift between the horizontal and vertical directions and vice versa. For this reason, we will introduce this firstdiscovered, to the best of our knowledge, method briefly in the following part.

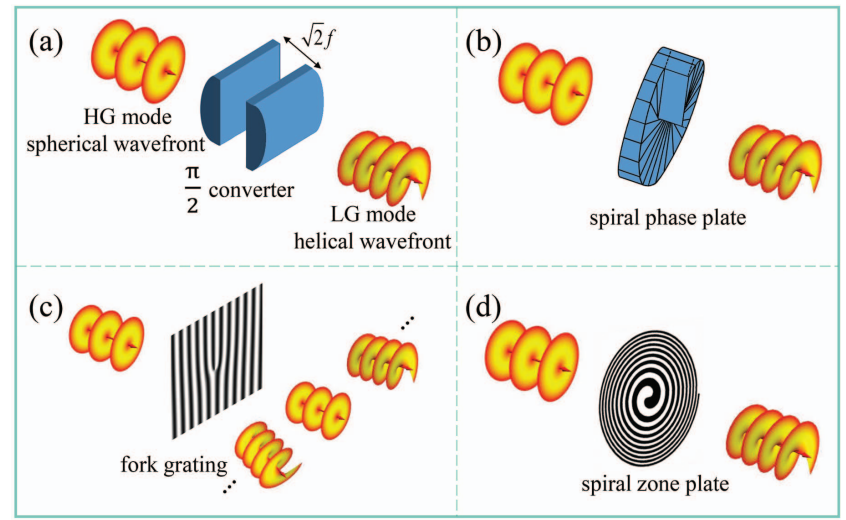

Fig. 2. Schematic diagrams of (a) $\frac{\pi}{2}$ converter, (b) SPP, (c) fork grating hologram, and (d) spiral zone plate with topological charges of $I=1$.

In order to obtain beams carrying OAM, it is necessary to introduce a phase difference of $\pi / 2$ in two vertical directions for the conversion between the HG and LG modes. As shown in Fig. 2(a), the $\pi / 2$ converter consists of two identical cylindrical lenses. Specifically, cylindrical lenses placed at the distance of $\sqrt{2} f$ and $2 f$ are known as $\pi / 2$ and $\pi$ converters, respectively. The LG mode with azimuthal index $l=n-m$ and radial index $p=\min (n, m)$ can be generated by passing an HG mode with indices $m$ and $n$ through a $\pi / 2$ mode converter. Despite that, optical vortices can be produced as such, the HG mode of high order is still required, and a complex optical system is inevitable. Moreover, in the case of an illuminating LG beam, the $\pi$ converter can reverse the sign of topological charge.

\subsubsection{Spiral phase plate}

It is well known that phase modulation can be achieved through spatial variations of thickness or refractive index. In this section, we will introduce the SPP, as it is one of the most commonly used refractive optical elements, which is usually considered to be the basis of various other alternative phase-modulation methods. As shown in Fig. 2(b), SPP is a transparent plate with a spiral thickness profile, where the optical height is proportional to the azimuthal angle ${ }^{[13,14]}$. With spiral phase retardation, one can note that optical beams with a helical wavefront can be directly transformed by an SPP.

One of the prominent attributes of generating a helical beam by SPP is that the conversion efficiency is extremely high and can almost reach $100 \%$. The SPP alleviates restriction on the incident light, for instance, a glass SPP can take high-power laser illumination without the requirement of an HG laser source or changing the direction of propagation. Nevertheless, since a supremely high precision is required for the manufacturing technique, the imperfection is also obvious due to the fact that the fabrication of the SPP with perfectly smooth variation in height is unattainable. Therefore, in most cases, approximate versions are manufactured in the implementation, where the smooth surface is replaced by many discrete steps ${ }^{[15]}$. As such, the performance of the fabricated SPP can be affected. More 
importantly, a specific SPP is only for the generation of optical vortices with specific topological charge, and it is rather sensitive to the wavelength of the incident light.

Based on the aforementioned drawbacks, many approaches have been proposed over the past few decades. For instance, an adjustable SPP can achieve various values of topological charges and be used in a wide range of wavelength at the same time $^{[16]}$. Recently, a flat SPP was introduced, the height of which remains unchanged, whereas the refractive index increases with the azimuthal angle ${ }^{[17]}$. Note that the accuracy of the generated optical vortices increases with an increasing number of phase steps. To simplify the fabrication procedure to the minimum, a recent study shows that only three phase steps are sufficient to generate the OAM mode accurately instead of a continuous variation using the method of modal decomposition ${ }^{[18]}$.

\subsubsection{Computer-generated holograms}

Holography is an approach to record and reconstruct the intensity as well as the phase of the optical field. Holographic optical elements such as fork grating and spiral zone plate are methods commonly used to generate optical vortices. It was Soskin et al. who first, to the best of our knowledge, proposed the concept of the fork grating ${ }^{[19-21]}$. The $l$-pronged fork dislocations indicate the topological charge $l$, and the reversed direction presents an opposite sign. In order to reconstruct the desired light, the interference pattern of a tilted plane light with complex amplitude of $\Phi_{1}(x, y)=A_{1}(x, y) \exp [i \phi(x, y)]$ and a vortex light with complex amplitude of $\Phi_{2}(x, y)=A_{2}(x, y) \exp (i l \theta)$ should be obtained as the first step. After interference, the intensity is

$$
\begin{aligned}
I= & B_{1}(x, y)+B_{2}(x, y) \exp \{i[\phi(x, y)-l \theta]\} \\
& +B_{2}(x, y) \exp \{-i[\phi(x, y)-l \theta]\},
\end{aligned}
$$

where $B_{1}(x, y)=\left|A_{1}(x, y)\right|^{2}+\left|A_{2}(x, y)\right|^{2}$ and $B_{2}(x, y)=A_{1}(x, y)$. $A_{2}(x, y)$. Then, binarize the fork-shaped grating, and record it on a photographic film using a lithography technique. Supposing $\Phi_{1}(x, y)$ as the reference wave, the following beams can be generated: $B_{1}(x, y) \exp [i \phi(x, y)]+B_{2}(x, y) \exp \{i[2 \phi(x, y)-l \theta]\}+$ $B_{2}(x, y) \exp (i l \theta)$, where the first term represents an unmodulated plane wave, the second term is the combination of a vortex wave and a reference wave, and the third term is the desired vortex wave. Note that optical vortices with varying topological charges can be produced by a single fork grating, while the efficiency can be different. Figure 2(c) shows that optical vortex beams are distributed at different transverse locations with different orders of diffraction.

Apart from the fork grating, the spiral zone plate is another computer-generated hologram that is mostly used to generate vortex beams ${ }^{[22,23]}$. In a cylindrical coordinate system $(r, \phi, z)$, the spherical vortex beam can be simply expressed as the spherical wave multiplied by the azimuthal term ${ }^{[24]}$,

$$
\Phi_{\mathrm{SVW}} \propto \frac{\exp \left[2 \pi i k \sqrt{r^{2}+\left(z-z_{1}\right)^{2}}\right]}{\sqrt{r^{2}+\left(z-z_{1}\right)^{2}}} \exp (2 \pi i l \phi),
$$

where the center of the spherical wave is $r=0$ and $z=z_{1}$. The hologram of a reference plane wave constructed with this spherical vortex beam can be expressed as

$$
I_{\text {holo }}=\left|\frac{\exp \left[2 \pi i k \sqrt{r^{2}+\left(z-z_{1}\right)^{2}}\right]}{r^{2}+\left(z-z_{1}\right)^{2}} \exp (2 \pi i l \phi)+1\right|^{2} \text {. }
$$

Particularly, the spiral zone plate can be degraded to a regular Fresnel zone plate with $l=0$. In practice, these computer-generated holograms are normally binarized for the fabrication of masks that can be given as follows:

$$
b(r)= \begin{cases}1, & 0 \leq \sqrt{L^{2}+r_{n}^{2}}-\sqrt{L^{2}+r^{2}}<\frac{\lambda}{2}, \\ 0, & \frac{\lambda}{2} \leq \sqrt{L^{2}+r_{n}^{2}}-\sqrt{L^{2}+r^{2}}<\lambda\end{cases}
$$

where $L$ is the focal length, and $r_{n}$ is the innermost radius of the $n$th zone. Figure 2 (d) shows the generation of a vortex beam with the spiral zone plate. Moreover, a series of vortex beams can be produced by a single micro-fabricated spiral zone plate at different positions along the propagating direction. The topological charge of the $n$ th-order vortex beam can be $n$ times larger than that of the first-order vortex beam. An electron vortex beam with a topological charge of 90 was observed in a higher diffraction order.

The difference between the fork grating and the spiral zone plate that comes in the beam to generate the hologram is either a plane wave or a spherical wave. As mentioned above, refractive SPP increases the topological charges with an increasing thickness, whereas in the computer-generated holograms, only more lines in the pronged dislocation or spirals are required to be increased. Therefore, especially in the case of higher values of topological charges, the fabrication of computer-generated holograms can be much easier than that of SPP.

\subsection{Using digital devices}

As introduced above, optical vortices can be generated by holographic approaches such as a fork-shaped grating and a spiral zone plate. However, an optical element can only generate an optical vortex beam with specific topological charge, which is inconvenient in experiment. What makes such methods particularly attractive is the digital devices such as a commercial SLM that typically include liquid crystal displays, positive transparency film, and a DMD. The SLM consists of substantial liquid crystal molecules independently modulated by corresponding applied voltage. SLM is an electro-optical device that enables us to manipulate the amplitude and phase of the light field, which can be classified into two types as reflective SLM based on liquid crystal on silicon and transmissive SLM based on transparent liquid crystal displays ${ }^{[25]}$. As such, vortex beams 
can be generated and detected by computer-generated holograms encoded on SLMs. Figure 3(a1) shows the schematic diagram of the experimental apparatus for multiplexing and de-multiplexing OAM modes for free-space communication, in which one SLM is used for mode creation, and the other is used for mode detection ${ }^{[26]}$. Figure 3(a2) shows phase holograms and corresponding far-field diffraction patterns under plane wave illumination. In Fig. 3(a3), optical vortex beams were generated, and the $l=0$ component denotes the correct alignment of the system. Compared with static optical elements, the modulation by the use of SLM can be achieved in real time, which greatly simplifies the experimental process, while the quality of the generated vortex beam is influenced by the number of pixels.

$\mathrm{DMD}$, as another candidate to generate vortex beams, is able to control incident light with high frame rate and a great number of spatial degrees of freedom. DMD is composed of tremendous high-speed digital switches such as aluminum reflective mirrors that can be modulated independently pixel by pixel. The resolution is determined by the number of pixels, and a small number of pixels may cause the quantization errors in encoding intensity and phase, and thus a larger number of micro-mirrors is preferable for high-quality beam generation. Binary data are sent to the static random access memory of DMDs and produce an electrostatic charge distribution, causing the deflection angle of each individual micro-mirror to be either -12 or +12 degrees, corresponding to "off" or "on" state. Beam shaping can be realized by switching the micro-mirrors on and off rapidly. Compared with liquid crystal displays absorbing up to $90 \%$ of the incident light with low transmittance and contrast, the DMD is a reflective SLM that can improve the image quality with higher throughput and superior diffraction efficiency ${ }^{[27]}$. Normally, owing to the viscosity of the liquid crystal, techniques based on liquid crystal SLM are limited by the switching rate. As an alternative beam shaping method, the DMD enables binary control (on or off) of the beams, the switching rate of which is extremely high in excess of $20 \mathrm{kHz}$, circa two orders faster than that of typical phase only SLMs ${ }^{[28]}$.

Similarly, optical vortices can be dynamically generated by DMD with spatially controllable phase and intensity. One of the most commonly used techniques for phase modulation by use of DMD is Lee holography ${ }^{[29]}$. For low resolution fields, it was shown that Lee holography with pixel dithering generated LG modes with the purity above $94 \%^{[30]}$. In 2010 , Ren et al. successfully transformed Gaussian beams into LG beams with different topological charges through projecting fork-shaped gratings on the $\mathrm{DMD}^{[31]}$. Later in 2013, Mirhosseini et al. proposed a technique to dynamically encode both phase and amplitude onto the laser beam through a DMD, realizing generation of OAM modes with rapid switching ${ }^{[32]}$. Figure 3 (b1) shows the experimental setup for generating perfect vortex beams by the use of amplitude DMD. Figure 3(b2) shows the intensity and phase mask pattern calculated with the Lee and superpixel methods, respectively. Figure 3(b3) represents the fidelity and efficiency comparison between those two common methods with topological charges from 1 to $100^{[33]}$.

Compared with conventional techniques, generating OAM modes by DMD makes it possible to switch modes at a very high speed with less optical elements. The superpixel method can independently control the amplitude and phase of light with high resolution by combining a spatial filter and a $\mathrm{DMD}^{[34]}$.

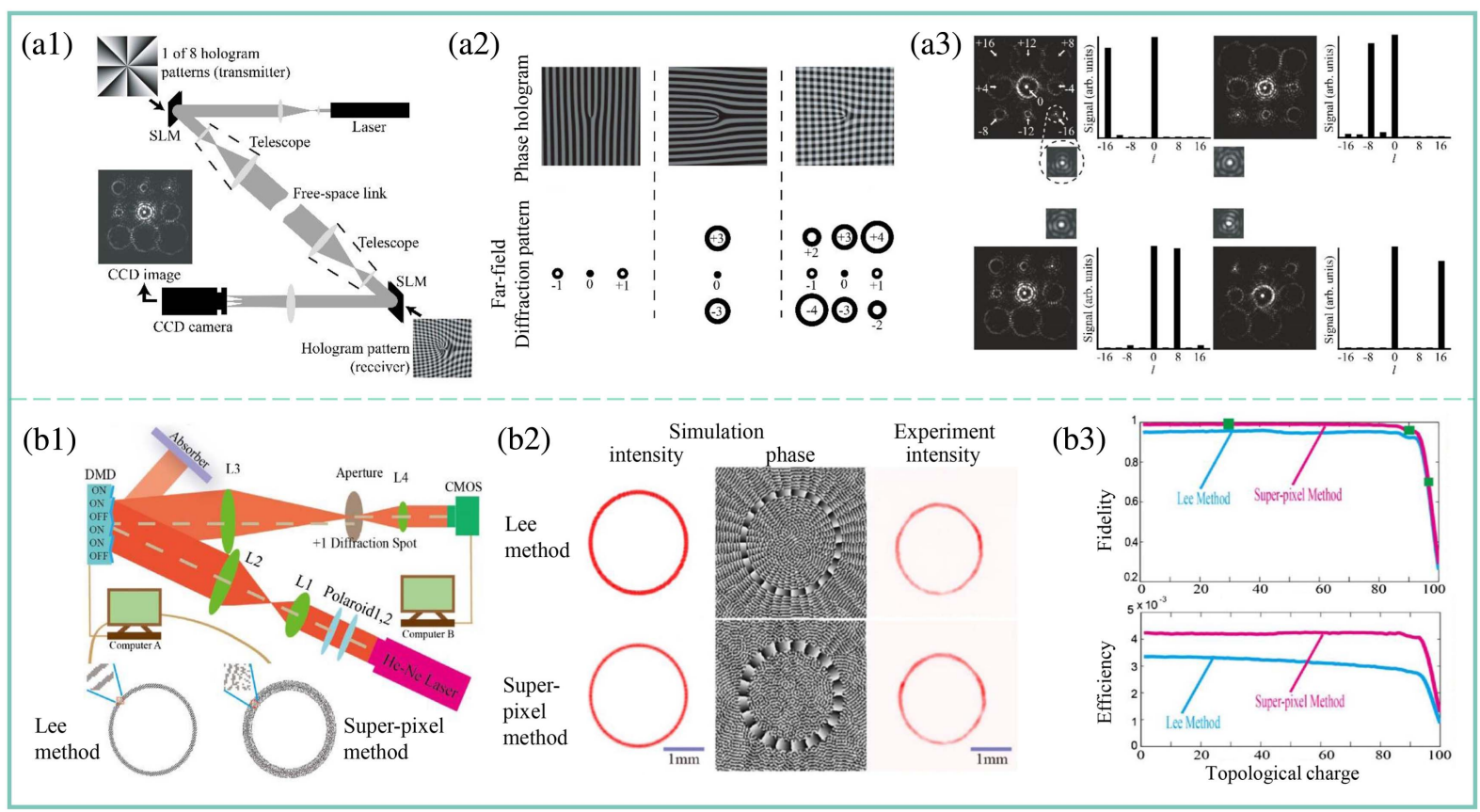

Fig. 3. Generation of optical vortex beam with digital devices: (a) SLM, (b) DMD. Schematic diagram of experimental apparatus of (a1) SLM and (b1) DMD. (a2) Phase holograms and corresponding far-field diffraction patterns. (a3) Generated vortex beams and OAM spectra ${ }^{[26]}$. (b2) Simulated and experimental results of vortex beams with $I=20$ by the Lee method and superpixel method, respectively. (b3) Fidelity and efficiency of the two methods ${ }^{[33]}$. 
By use of a clock signal to achieve the switching and load computer holograms onto the memory of the DMD, the quantum number of generated OAM modes can be switched at the speed of $4 \mathrm{kHz}$. Actually, for the commercially available DMD (TI DLP7000), the switching speed can be achieved as high as $32 \mathrm{kHz}$. More importantly, compared with the phase only SLM, the cost of the DMD can be much lower. However, it is noted that the efficiency of OAM modes generation is measured to be only $1.5 \%$, and the maximum theoretical efficiency of the binary amplitude grating is circa $10 \%$, whereas the efficiency of phase only SLMs is usually above $50 \%$ and can even reach $90 \%$. Compared with the Lee holography method, the fidelity and efficiency of the superpixel method can be improved.

\subsection{Using photon sieves}

Apart from the aforementioned methods, a binary amplitude mask is usually used to shape both the phase and amplitude of lights; one example is the photon sieve composed of a large number of pinholes that can tightly focus the incident light. In 2001, Kipp et al. proposed a photon sieve with transmissive pinholes with designed diameters and distribution in order to overcome the limitation in spatial resolution, reduce unwanted diffraction orders, and further eliminate the scattered intensity by the conventional zone plate ${ }^{[35]}$. A prominent attribute is that the photon sieve is simple to be manufactured due to its self-supporting structure in the design. Originally, the photon sieve is an optical diffractive element that consists of annularly distributed pinholes based on the Fresnel zones to improve the spatial resolution and focusing ability for soft X-rays. Limitations using conventional zone plates can be overcome by pinholes with varying diameters rather than concentric rings as basic diffracting elements. Owing to the non-absorbent characteristic, the photon sieve can be used in a broad spectrum ranging from far infrared to visible light, extreme ultraviolet, and X-rays. The light field passing through the photon sieve is controlled by the parameters of holes such as their position, number, and size. The focal length of the photon sieve is inversely proportional to the wavelength so the chromatic aberration can be obvious. The diameter of the outermost ring will be smaller with a larger radius, and thus higher resolution can be achieved.

In 2015, Qiu et al. proposed an ultrahigh-capacity photon sieve containing subwavelength holes arranged in two structural orders of aperiodicity and randomness ${ }^{[36]}$. The aperiodic photon sieve composed of 7240 holes with varying size is used to realize sub-diffraction-limit focal spot (full width at half-maximum $\sim 0.32 \lambda$ ), and the random photon sieve acts as an uniform optical hologram with high diffraction efficiency. Such a hybrid method combining the aperiodic and random photon sieve is viable for manipulation of the polarization, amplitude, and phase of the visible light aiming at superfocusing and high-quality holograms. Figure 4(a1) shows the schematic view of the spiral photon sieve, and the concept of photon sieves was extended to spiral photon sieves for the generation of the hard X-ray vortex beams ${ }^{[37]}$. As illustrated in Figs. 4(a2) and 4(a3), vortex beams with topological charge of $l=1$ were generated by the

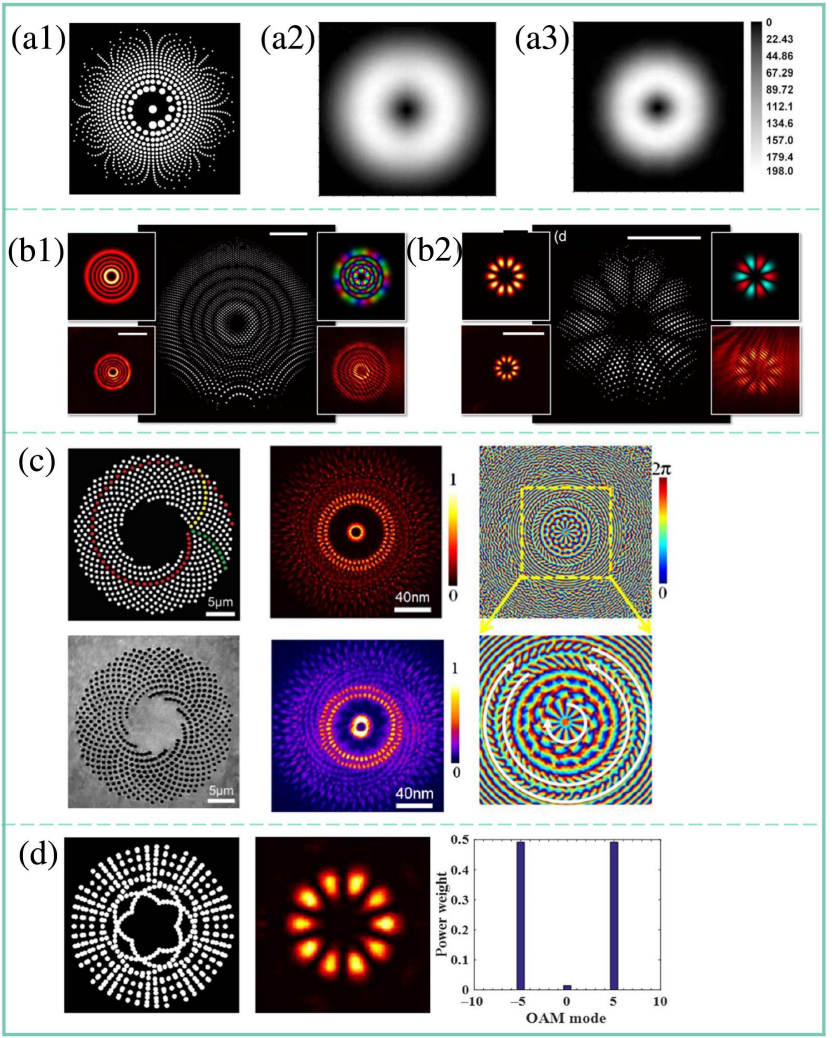

Fig. 4. (a1) Schematic view of the spiral photon sieve. Vortex beams generated by (a2) spiral zone plate and (a3) spiral photon sieve ${ }^{[37]}$. (b) Target intensity and phase (top left and right) and experimentally measured intensity and plane wave interference pattern (bottom left and right) of (b1) the $L G$ beam with $I=5, p=4$ and (b2) superposition of two LG beams with $I= \pm 5, p=0^{[38]}$. (c) Electron vortex beam generated by rotationally symmetric mask based on Archimedean spirals $^{[39]}$. (d) Schematics of simulated mask, intensity profile, and OAM spectrum (left to right) for the generation of photonic gear with $l= \pm 5^{[40]}$

use of a spiral zone plate and spiral photon sieve, respectively. One can note that the spiral photon sieve has lower background noise and better spatial resolution than the spiral zone plate under specified conditions. Moreover, Liu et al. demonstrated generalized photon sieves based on the photon sieve first, to the best of our knowledge, introduced by Kipp et al. in 2001 as a generalization to generate an arbitrary structured complex field by simple pinhole arrays. Compared with the original photon sieve, the designed generalized photon sieve consists of only a few hundred instead of thousands of pinholes to create structured beams at the focus. Figure 4(b) shows the photon sieves (middle), target field (top), and experimentally measured fields (bottom) for the generation of LG beams with $l=5, p=4$ [Fig. 4(b1)] and the superposition of two LG beams with $l= \pm 5$, $p=0$ [Fig. 4(b2)], in which the distribution of pinholes is similar to the required amplitude of the target beam itself ${ }^{[38]}$.

Similarly, vortex beams can be produced by the use of electron sieves. In 2017, Yang et al. proposed the electron sieves with a rotationally symmetric structure to flexibly and systematically generate the electron vortex beams ${ }^{[39]}$. Figure $4(\mathrm{c})$ exhibits the 
electron sieve, intensity, and phase distribution (left to right) of the generated vortex beam using a rotationally symmetric mask based on Archimedean spirals. Different from spiral computergenerated holograms, where different OAM modes are focused at different on-axis positions, while others exist only in the background, this kind of electron sieve can generate compound vortex beams with three bright rings $(l=-11,44$, and 55$)$. Later in 2019 , the same research group further produced an optical vortex beam with unique OAM modes by a simple pinhole plate ${ }^{[40]}$. Also, superposition of OAM modes with opposite topological charges, known as photonic gears, is generated using pinhole plates. Suppose that the pinhole plate is composed of several spirals; the radial and azimuthal expressions of the $n$th pinhole in the $m$ th spiral are $r_{n}= \pm\left(\frac{l z \lambda \alpha_{n}}{\pi}+r_{0}^{2}\right)^{1 / 2}$ and $\alpha_{m, n}= \pm\left(\frac{2 \pi n}{N}+\frac{2 \pi m}{M}\right)$, respectively. As shown in Fig. 4(d), photonic gear with $l= \pm 5$ is generated by a pinhole plate, and the intensity pattern exhibits a $2 l$ petal-like structure as expected. The OAM mode spectrum shows that the purity of generated OAM modes is fairly high.

\subsection{Using metasurfaces}

In traditional methods, changing phase by accumulating the propagation distance makes it very bulky to generate optical vortices and restrains further development in integrated optics, and thus elements with smaller size are imperative to generate optical vortex beams for the OAM-based applications at miniature scales. Metasurface, an equivalent two-dimensional device, is constructed by artificial arrays of meta-atoms etched on the ultrathin plate that can control the phase, amplitude, and polarization state arbitrarily. The incident light can be imparted with a space-variant abrupt phase change through an ultrathin metasurface instead of acquiring the desired phase change via the propagation effect. The metasurface revolutionized the concept of light manipulation and paved the way to the construction of ultrathin optical devices.

Two primary approaches to design the metasurfaces are based on Pancharatnam-Berry (PB)/geometry phase, dynamic phase, and detour phase ${ }^{[41-44]}$. A metasurface using geometry phase is composed of anisotropic subwavelength nanoantennas with spatially varying orientation that can tailor the light field pixel by pixel. As shown in Fig. 5(a), focused perfect vortex beams with annular ring intensity independent of topological charges were generated by the metasurfaces consisting of rectangularhole nanoantennas combining the phase profiles of the SPP, Fourier transform lens, and axicon based on the geometry phase ${ }^{[45]}$. The principle advantage of this type of metasurface is the independence of the inherent dispersion; thus it can be used in a wide range of spectra. Considering that the incident light is right-handed circularly polarized with a normalized Jones vector $1 / \sqrt{2}[1, i]$, transmittance light passing through the metasurface can be decomposed into two components including a polarization-residual term with the original polarization state and a polarization-converted term with an orthogonal polarization state, the electric field of which can be expressed as ${ }^{[46]}$

$$
\begin{aligned}
\vec{E}_{\mathrm{out}}^{\mathrm{RCP}} & =\vec{E}_{\mathrm{con}}^{\mathrm{RCP}}+\vec{E}_{\mathrm{res}}^{\mathrm{RCP}} \\
& =\frac{1}{\sqrt{2}}\left(\frac{A}{A+B} e^{i\left(\frac{\pi}{2}-2 \phi\right)}\left[\begin{array}{c}
1 \\
-i
\end{array}\right]+\frac{B}{A+B}\left[\begin{array}{l}
1 \\
i
\end{array}\right]\right),
\end{aligned}
$$

where $A$ and $B$ represent amplitudes of the converted and residual parts, and $\vec{E}_{\text {con }}^{\mathrm{RCP}}$ and $\vec{E}_{\text {res }}^{\mathrm{RCP}}$ denote the electric field of the converted and residual terms, respectively. One can note from the expression that there is an abrupt phase change in the converted part due to the spin-orbit transformation in anisotropic and inhomogeneous media, whereas there is no phase delay in the residual part. Moreover, the polarization-converted term can be imparted with an phase shift variation $2 \phi$ with a spatial orientation angle $\phi$.

Other than the geometry phase, another dominant approach to design a metasurface is modifying the transmission phase by changing the meta-atoms, known as dynamic phase. The geometries of meta-atoms can be any shape ranging from cross slits $^{[47,48]}$, nanofin ${ }^{[49]}$, and $\mathrm{u}$-shaped slot ${ }^{[50]}$ to $\mathrm{v}$-shaped antenna $^{[51,52]}$, etc. A typical metasurface based on dynamic phase is shown in Fig. 5(b), where different sizes of nanoholes are distributed in eight fan-shaped sections, realizing the phase tuning of $(0,2 \pi)^{[53]}$. However, owing to the strong absorption in metallic materials, metasurfaces etched on a metallic structure suffer much loss in the visible spectrum, whereas it can be used in the higher wavelength spectrum such as infrared light.

Moreover, vortex beams can be generated by metasurfaces based on the combination of dynamic and geometry phase. Figure 5(c) shows a metasurface as a $J$ plate composed of rectangular-shaped nanoantennas with different sizes, which coverts right and left circular polarizations into states with independent values of $\mathrm{OAM}^{[54]}$. Consider that two orthogonal polarization states can be expressed in the linear polarization basis $\left|\lambda^{+}\right\rangle=$ $\left[\begin{array}{c}\cos \chi \\ e^{i \sigma} \sin \chi\end{array}\right],\left|\lambda^{-}\right\rangle=\left[\begin{array}{c}-\sin \chi \\ e^{i \sigma} \cos \chi\end{array}\right]$, where $\sigma$ and $\chi$ are related to the polarization states. The Jones matrix for the $J$ plate can be written as

$$
J(\phi)=e^{i \sigma}\left[\begin{array}{cc}
e^{i \sigma} \mu_{1}(\phi) & \frac{\sin 2 \chi}{2} \nu(\phi) \\
\frac{\sin 2 \chi}{2} \nu(\phi) & e^{-i \sigma} \mu_{2}(\phi)
\end{array}\right],
$$

where $\phi$ is the azimuthal angle, $n$ and $m$ are integers that control the output OAM, $\mu_{1}(\phi)=e^{i m \phi} \cos ^{2} \chi+e^{i n \phi} \sin ^{2} \chi, \quad \nu(\phi)=$ $e^{i m \phi}-e^{i n \phi}$, and $\mu_{2}(\phi)=e^{i m \phi} \sin ^{2} \chi+e^{i n \phi} \cos ^{2} \chi$. Thus, it can be found that $J(\phi)\left|\lambda^{+}\right\rangle=e^{i m \phi}\left|\left(\lambda^{+}\right)^{*}\right\rangle$ and $J(\phi)\left|\lambda^{-}\right\rangle=$ $e^{\text {in } \phi}\left|\left(\lambda^{-}\right)^{*}\right\rangle$, as illustrated in Fig. 5(c), where light with spin state $\left|\lambda^{+}\right\rangle\left(\left|\lambda^{-}\right\rangle\right)$illuminates on the $J$ plate, and a vortex beam with topological charge of $m(n)$ can be generated with flipped handedness.

Except for generation of a single vortex beam, as illustrated in Fig. 5(d), metasurfaces composed of three parts can generate multiple optical vortex beams at different focal planes, combining the functionalities of a lens and an $\mathrm{SPP}^{[55]}$. Nevertheless, each approach has its own limitations for the generation of vortex beams. For metasurfaces based on geometric phase, the purity of the generated vortex beam is sensitive to the dichroism 


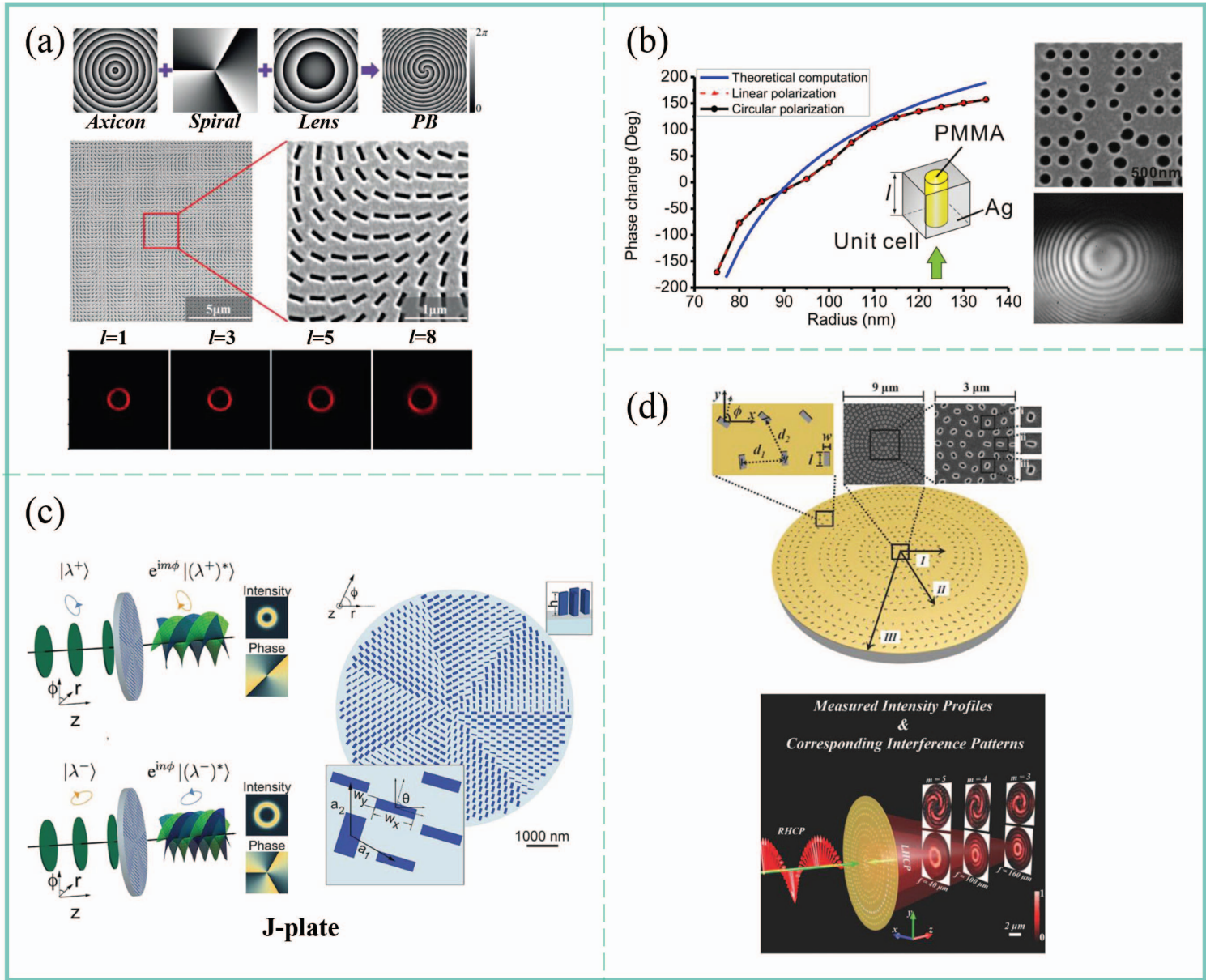

Fig. 5. Generation of optical vortices through metasurfaces based on (a) dynamic phase ${ }^{[45]}$, (b) geometry phase ${ }^{[53]}$, (c) the combination of dynamic and geometry phase ${ }^{[54]}$, and (d) optical vortex generator with multiple focal planes ${ }^{[55]}$.

of the material, especially for vortex beams with high-order topological charges. The metasurface has fundamentally revolutionized the generation and manipulation of optical vortex beams, which has potential in the fields of optical communications, integrated optics, etc.

\subsection{Using other techniques}

Most traditional devices can be damaged with high-power laser illumination; hence approaches such as the foil-made light fan and $s$-plate were introduced to produce optical vortex beams ${ }^{[56,57]}$. Optical retarder elements with azimuthal rotation of principle axes such as $s$-plate and $q$-plate have attracted much attention. The $s$-plate is a superstructured half-wave plate polarization converter that can be used to produce optical vortices and beams with azimuthal/radial polarization ${ }^{[58]}$. Liquid crystal $q$ plate, as a spatially non-uniform birefringent optical element, has been researched due to its ability to manipulate both the SAM and OAM of photons ${ }^{[59]}$. The $q$-plate is a birefringent device that consists of liquid crystal retarders, the optical axes of which are rotated with respect to the center of the device, and the retardance of each retarder can be manipulated by the applied voltage. Defining the direction at the $x$ axis as $\phi_{0}$, the rotation angle of the liquid crystal retarder can be expressed as $\alpha(r, \phi)=q \phi+\phi_{0}$, where $q$ is the topological charge of the $q$-plate.

Similar to the half- and quarter-wave plate, the Jones matrix of a $q$-plate can be written as ${ }^{[60]}$

$$
J_{q}=\left[\begin{array}{cc}
\cos 2 \alpha & \sin 2 \alpha \\
\sin 2 \alpha & -\cos 2 \alpha
\end{array}\right]
$$

with the incidence of a right-handed circularly polarized light $E_{\text {in }}=E_{0} \times[1, i]$, and the resultant light field can be expressed as

$$
\begin{aligned}
E_{\text {out }} & =J_{q} E_{\text {in }}=E_{0}\left[\begin{array}{cc}
\cos 2 \alpha & \sin 2 \alpha \\
\sin 2 \alpha & -\cos 2 \alpha
\end{array}\right]\left[\begin{array}{l}
1 \\
i
\end{array}\right] \\
& =E_{0} \exp (i 2 q \phi) \exp \left(i 2 \phi_{0}\right)\left[\begin{array}{c}
1 \\
-i
\end{array}\right] .
\end{aligned}
$$

Noting that a vortex light field with an opposite circular polarization plus an azimuthal phase is transformed, the topological charge of which is $2 q$. Analogously, in the case of a left-handed circularly polarized incident light, the sign of the topological charge can be reversed, i.e., the output helical wavefront is determined by the incident polarization state. 
Despite the aforementioned optical elements known as passive methods, direct generation of vortex beams can also be achieved from the solid-state laser cavity by active methods, i.e., optical vortex lasers. Generally, techniques used to directly produce vortex beams from the laser resonator can be categorized as annular beam pumping, central damaged cavity mirror, thermally induced mode aperture in side pumped vortex laser, and computer-controlled vortex laser. The pump beam can be shaped into an annular beam through the capillary fiber in order to produce vortex output, where the sign of topological charge can be controlled by the intracavity etalon angle. Spatial intensity matching between gain volume in the laser resonator and $\mathrm{LG}_{0, l}$ modes can be achieved by annular beam pumping, realizing the generation of $\mathrm{LG}_{0, l}$ modes with high value of $l$ up to $200^{[61]}$. Moreover, vortex beams can be generated by the cavity mirror with removed reflective surface, i.e., a central damage spot $^{[62]}$. Oscillation of the LG mode can be produced with suppressed lower-order Gaussian mode after passing through the damage spot, the size of which is supposed to be well matched to the resonator mode size. Also, vortex beams can be directly generated through the laser system without the need of any additional beam shaping elements such as an annular pump beam and the damaged mirror introduced above ${ }^{[63]}$. Such a laser system employing the $\mathrm{Nd}: \mathrm{YVO}_{4}$ slab crystal can efficiently suppress the Gaussian mode and produce a preferential vortex mode once the pump level is beyond the threshold, resulting in the generation of vortex beams. In addition, applying SLM to the laser cavity makes it possible to flexibly tailor vortex beams with an arbitrary phase and intensity profile, in which an SLM acts as the resonator mirror. It was demonstrated that laser modes including Airy beams, $\mathrm{HG}$, and higher-order $\mathrm{LG}_{p, l}$ modes were successfully generated by a computer-based laser system. Due to the optical damage of the SLM, the power scaling of such systems is still limited ${ }^{[64]}$.

Moreover, despite optical vortex beams or electron vortex beams, plasmonic waves can possess OAM as well, known as a plasmonic vortex. Surface plasmon polaritons are collective oscillation of incident electromagnetic waves coupled with electrons on the metal surface confined to the interface that exhibit evanescence in the direction perpendicular to the interface. The energy of the plasmonic field is confined in a subwavelength scale that can be used to surmount the conventional diffraction limit and provide colossal local field intensity. To generate plasmonic vortices with specific topological charge, elements such as plasmonic metasurfaces and plasmonic vortex lenses have been researched ${ }^{[65]}$. It has been shown that plasmonic waves carrying well-defined OAM can be generated by illuminating incident lights to the metal surfaces with various subwavelength structures such as nanoslits ${ }^{[66]}$, nanoholes ${ }^{[67-69]}$, and gratings ${ }^{[70]}$.

\section{Detection of OAM}

Vortex beams have been widely used in the fields of optical communication $^{[6,7]}$, optical trapping ${ }^{[71,72]}$, quantum information $^{[73,74]}$, etc. Therefore, it is imperative to measure the topological charges of vortex beams. In this section, various methods for the detection of OAM are reviewed based on interference and diffraction method, geometric coordinate transformation, deep learning, and surface plasmon polaritons.

\subsection{Using interference method}

There are many methods to measure the OAM of vortex beams by the principle of interference, among which the most basic one is interfering with a tilted plane wave so that a fork-shaped interference pattern can be exhibited ${ }^{[22]}$. In this case, as introduced in Section 2, the interference pattern is similar to the fork grating with $l$-dislocation fringes, the direction of which is dependent on the sign of the corresponding topological charge, and thus the OAM of the vortex beam can be effectively detected. Moreover, when the vortex beam interferes with a coaxial plane wave, it can be expected to be a petal-like interference pattern, the number of which is $|l|$ due to its spiral phase factor $\exp (i l \phi)$. Interfering with the spherical wave instead of the plane wave, the interference pattern will be helical fringes, and the number of fringes is also $l^{[75,76]}$. Also, the vortex beam can be measured by interfering with its mirror image ${ }^{[77,78]}$. When the vortex beam with topological charge $l$ is reflected by a mirror, the rotation direction of the reflected beam can be reversed with the topological charge of $-l$. Then, interfering the vortex beam with its mirror image, a $2 l$ petal-like interference pattern can be obtained.

The OAM of the vortex beam can be detected by the use of double-slit interference ${ }^{[79]}$. Figure 6(a) shows the phase distribution of a vortex beam with $l=1$, in which the azimuthal angle varies from 0 to $2 \pi$ in one cycle. As such, after passing through two slits, one can note that the phase variation between the left and right slits is also $0-2 \pi$, resulting in additional phase difference along the $y$ direction. Meanwhile, the optical path length difference between the observation spot and slits remains the same, and thus the additional phase that only comes from the azimuthal phase variance along the wavefront is added to the interference intensity distribution. As a consequence, the phase change leads to the shift of the interference fringe in the $x$ direction, the number of which indicates the topological charge of incident light, and the corresponding sign can be identified by the direction of fringe. The double-slit interference method is easy to realize with a simple optical element, and the value of the topological charge can be identified merely with naked eyes ${ }^{[80]}$. This method can be further applied in the case of partially coherent beams ${ }^{[81]}$, single photons ${ }^{[82]}$, etc.

In addition, other methods have been proposed to detect the OAM of vortex beams such as dynamic angular double slits ${ }^{[83-85]}$ and multipoint interferometer ${ }^{[86,87]}$. When a vortex beam with topological charge of $l$ is vertically incident on the dynamic angular double slits, light can only pass through two slits, as shown in Fig. 6(b). The interference intensity can be converted to the voltage by a photoelectric detector, where the voltage is maximum with the constructive interference and vice versa. It can be observed that the received signals are periodic, and the number of peaks equals the absolute value of topological charge except the first split peak. Due to its continuous scanning, 


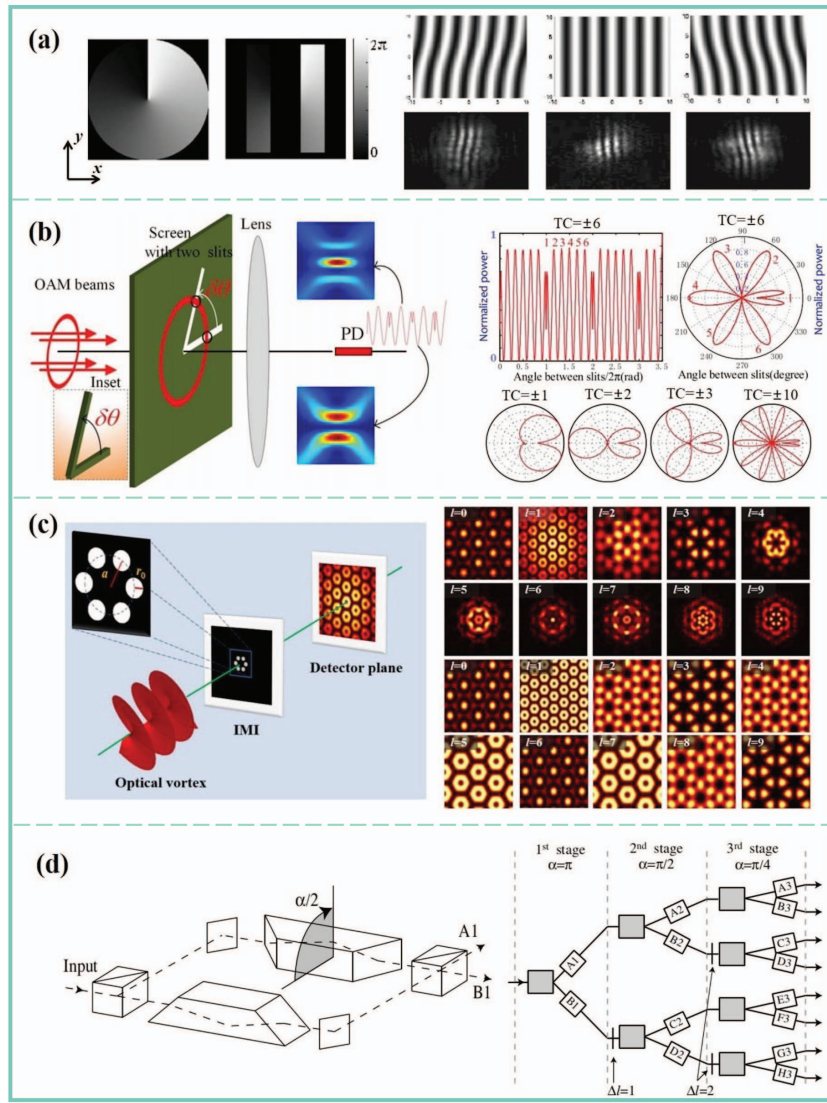

Fig. 6. (a) Left to right: phase distribution of a vortex beam with $/=1$, the spiral phase passing through the double slits, and interference intensity distribution ${ }^{[79]}$. Schematic and results of (b) angular double-slits interference

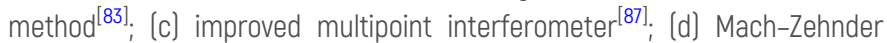
interferometer with a rotating Dove prism and cascading interferometers ${ }^{[89]}$.

the dynamic angular double-slits interference can be used to detect OAM modes with larger topological charge. Moreover, a multipoint interferometer composed of uniformly distributed circular holes can discriminate the topological charges of incident vortex beams based on different interference patterns ${ }^{[86]}$. However, it is only available for vortex beams with lower OAM, because the interference patterns will repeat periodically with higher topological charges. Based on these defects, an improved multipoint interferometer with larger pinholes was proposed, as shown in Fig. 6(c). Because larger pinholes make it possible to get more information of incident beams, the improved multipoint interferometer can distinguish vortex beams with higher $\mathrm{OAM}^{[87]}$. Intensity patterns for vortex beams with varying OAM modes by the use of a multipoint interferometer (upper) and improved multipoint interferometer (lower) are exhibited, respectively.

The Mach-Zehnder interferometer with Dove prisms can also be used to measure OAM states of vortex beams ${ }^{[88,89]}$. When the Dove prism rotates $\psi / 2$ along the long axis, the phase of the incident beam will rotate $\psi$ after passing through the Dove prism. Assuming that the incident light is a vortex beam carrying topological charge of $l$, the phase difference of $\Delta=l \psi$ can be generated between beams along two arms. On this basis, Leach $e t$ al. proposed a method to effectively distinguish a single-photon OAM mode, where photons with odd and even $l$ can be divided into two different outputs ${ }^{[89]}$. As shown in Fig. 6(d), arbitrary OAM modes can be measured by cascading additional Mach-Zehnder interferometers with different rotation angles. Moreover, methods such as a Sagnac interferometer were proposed to measure the topological charge with better stability $^{[00,91]}$.

\subsection{Using diffraction method}

Typically, detection of OAM based on diffraction can be divided into the aperture diffraction method ${ }^{[92-95]}$, grating diffraction method $^{[26,96,97]}$, cylindrical lenses ${ }^{[98,99]}$, etc. The vortex beam is incident on a triangle or square aperture, and the value of its topological charge can be detected from the diffraction pattern in the far field. The aperture diffraction method was firstly, to the best of our knowledge, proposed by Hickmann et al. in $2010^{[92]}$. As shown in Fig. 7(a), the diffraction pattern of a vortex beam passing through an equilateral triangular aperture can reflect its topological charge $l$. To be specific, the topological charge of the incident vortex beam is $l=N-1$, where $N$ is the number of points on any side of the triangular lattice array. The sign of topological charge $l$ can be distinguished by the rotation direction of the diffraction pattern. Similarly, the far-field diffraction intensity distribution is also a triangular lattice array after passing through the annular triangle aperture, where the diffraction intensity distribution becomes clearer with a higher ratio of inner/outer apertures ${ }^{[94]}$. For the square aperture, the lattice array is square, and the absolute value of the topological charge can be detected similarly, whereas it is unachievable to

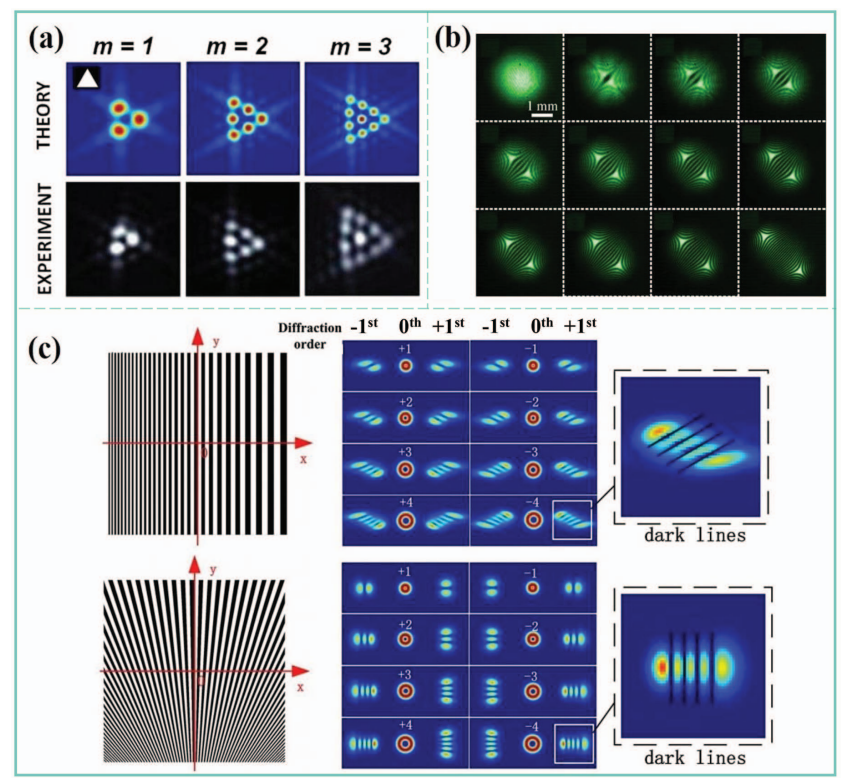

Fig. 7. (a) Diffraction patterns of triangular aperture ${ }^{[92]}$. (b) The intensity distribution of the vortex beam is measured by a cylindrical lens ${ }^{[99]}$. (c) Schematic and results of the gradually changing period grating ${ }^{[97]}$. 
distinguish the sign of topological charge ${ }^{[95]}$. When the topological charge of the incident beam is odd, the relationship between the number of dots and the topological charge can be expressed as $l=2 N-1$, otherwise $l=2 N-2$.

A cylindrical lens is a kind of aspheric lens that can effectively reduce spherical and chromatic aberration with the function of one-dimensional amplification. As mentioned in the second section, a mode converter consisting of a cylindrical lens can convert an HG beam into an LG beam and vice versa. Based on the theory, detection of OAM can be realized by the use of a cylindrical lens ${ }^{[98,99]}$. Since the optical-field distribution of the HG beam is an orderly arrangement of multiple light spots related to its order, an LG beam can be transformed into an HG mode by a cylindrical lens, and its topological charge $l$ can be directly identified based on the diffraction light field. Figure 7(b) shows the experimental results with incident vortex beams, where the topological charges are $0,1,2, \ldots, 10$, and 20 , respectively.

One can note that vortex beams can be generated using computer-generated holograms. A vortex beam with topological charge $l$ can be generated at the first diffraction order with a Gaussian beam passing through an $l$-dislocations fork-shaped grating. Similarly, a vortex beam with $-l$ can be transformed into a fundamental mode Gaussian beam by eliminating its helical phase through an $l$-dislocations fork-shaped grating, and thus the detection of OAM can be realized. However, this method is only appropriate for vortex beams with specific OAM, and multiple holograms are needed for the detection of different OAM modes, resulting in the low efficiency. Therefore, more complex holograms were proposed by superimposing multiple holograms based on the Daman grating, making it possible to measure multiple topological charges through a single grating ${ }^{[26]}$. However, the efficiency is still not high enough due to the energy of the incident beam being distributed to every output mode. After that, the gradually changing period grating was presented $^{[97]}$, as shown in Fig. $7(\mathrm{c})$. In the positive first-order diffraction pattern, the light spot is divided into stripes, the number of dark stripes is equal to the value of topological charge, and the direction of the light spot corresponds to its sign. Alignment is not neccessary in such a method, while the diffraction efficiency is relatively low so that it only reaches $10 \%$. Moreover, the annular grating is another kind of grating that can measure the vortex beams with fractional topological charge from 0.1 to 1 and higher-order topological charge as high as \pm 25 . Usually, the annular grating can be categorized as phase and amplitude gratings. Compared with the aforementioned gratings, an annular grating can realize detection with high efficiency and no alignment requirement ${ }^{[100]}$.

\subsection{Using geometric coordinate transformation}

Separation of OAM modes by the use of geometric coordinate transformation is also an effective method for vortex beams detection, the basis of which is the conversion from OAM into linear momentum ${ }^{[101,102]}$. In this method, diffractive optical elements convert the spiral phase related to the topological charge into transverse phase gradient, and the lens focuses input vortex beams with different topological charges to different transverse positions. As shown in Fig. 8(a), three SLMs are used to generate vortex beams (SLM1), convert the azimuth position in Cartesian coordinates to the transverse position in log-polar coordinates (SLM2), and correct the phase distortion introduced by the geometric coordinate transformation (SLM3), respectively. Optical lenses L1 and L2 are used for Fourier transformation and focusing the transformed lights. As such, OAM states of the vortex beam can be measured according to intensity distribution on the detection plane. This method can be used to identify the superposition of multiple vortex beams with different topological charges.

As introduced above, the log-polar transformation truncates the azimuthal light field of the vortex beam and transforms it into a plane wave with only a finite length, and thus focusing spots produced by vortex beams with adjacent topological charges overlap slightly due to the existence of the diffraction limit, causing mutual crosstalk. Based on this, Chen et al. proposed the concept of spiral transformation ${ }^{[103]}$. Different from log-polar transformation, the new helical coordinate transformation decomposes the vortex beam along the helical path and transforms it into an oblique plane wave mode, as shown in Fig. 8(b). As a result, more phase information of the vortex beam can be obtained in the plane wave after spiral transformation, and higher resolution can be achieved owing to smaller spots formed after focusing.

Log-polar transformation requires two phase elements for mode separation, and the alignment of these two elements is very strict. In order to improve the miniaturization of the optical system, more designs have been proposed, such as integration of

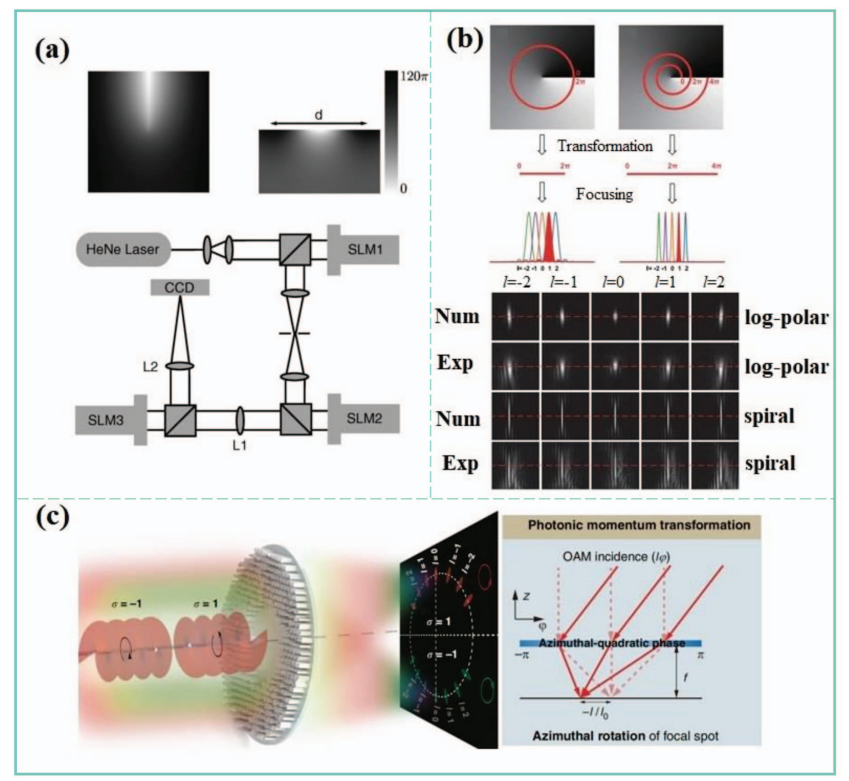

Fig. 8. (a) Phase profiles of the transforming and the phase-correcting optical element (top). Schematic of the optical system (bottom) ${ }^{[101]}$. (b) Schematic diagram and results of log-polar transformation and spiral transformation for OAM modes sorting ${ }^{[103]}$. (c) Schematic and results of the detection of SAM and OAM by photonic momentum transformation ${ }^{[104]}$. 
expansion and correction and non-paraxial log-polar transformation $^{[105-107]}$. Moreover, metasurfaces can be used for the detection of $\mathrm{OAM}^{[108,109]}$. A single azimuthal-quadratic phase metasurface that combines geometric phase and dynamic phase can realize coordinate transformation and focusing simultaneously $^{[104]}$. Such an optical device cannot be easily realized by either diffractive or refractive optics. Vortex beams with different topological charges can be transformed into focusing transverse plane modes by photonic momentum transformation. As shown in Fig. 8(c), the shape of focusing patterns can be almost neglected, and a rotation is exhibited along the circle. Also, the SAM can be detected due to the geometric phase being spindependent, and the phase profile will be reversed with an opposite spin direction. Such a method can also measure vector vortex beams with polarization and phase singularities and OAM modes with a certain interval step.

\subsection{Using deep learning}

Recently, deep learning has been widely used in the fields of image processing with its powerful advantages of data analysis and information processing by the use of local connection, shared weight, pooling, and multi-layer structure ${ }^{[110]}$. Typically, a neural network is composed of convolutional layers, pooling layers, and fully connected layers. The convolutional layer is used to detect the local combination of features from the previous layer, the pooling layer is used to merge semantically similar features into one, and the fully connected layer is used to realize the classification.

In the free-space communication system, atmospheric turbulence can cause wavefront distortion of the transmitted beams, which is a serious challenge for OAM-based communication systems. Owing to the rapid development of machine learning, especially deep learning technology, vortex beams detection based on the convolutional neural network (CNN) has gradually emerged in recent years ${ }^{[111-114]}$. Under different atmospheric turbulence, Li et al. proposed a multi-layer representation learning with high accuracy to recognize OAM modes of multiple vortex beams ${ }^{[112]}$, as shown in Fig. 9(a). At the transmitter, Gaussian lights are modulated into different modes of vortex beams and then transmitted through the atmospheric turbulence channel. At the receiver, the intensity images of vortex beams are collected. The received intensity images of vortex beams transmitted through different atmospheric turbulences are resized to speed up the training process, then the convolutional layers extract image features, the pooling layers compress the dimensions, and finally a fully connected layer output results. Later, Wang et al. designed a special six-layer neural network, which can effectively identify OAM modes with different turbulence intensity levels, transmission distances, and mode spacings under reasonable computational complexity and accuracy, as shown in Fig. 9(b) ${ }^{[113]}$. Moreover, Dedo et al. proposed a joint scheme of the Gaussian algorithm and CNN. After initial recovery of the vortex beams intensity distribution using the Gerchberg-Saxton (GS) algorithm, CNN classifies multiple

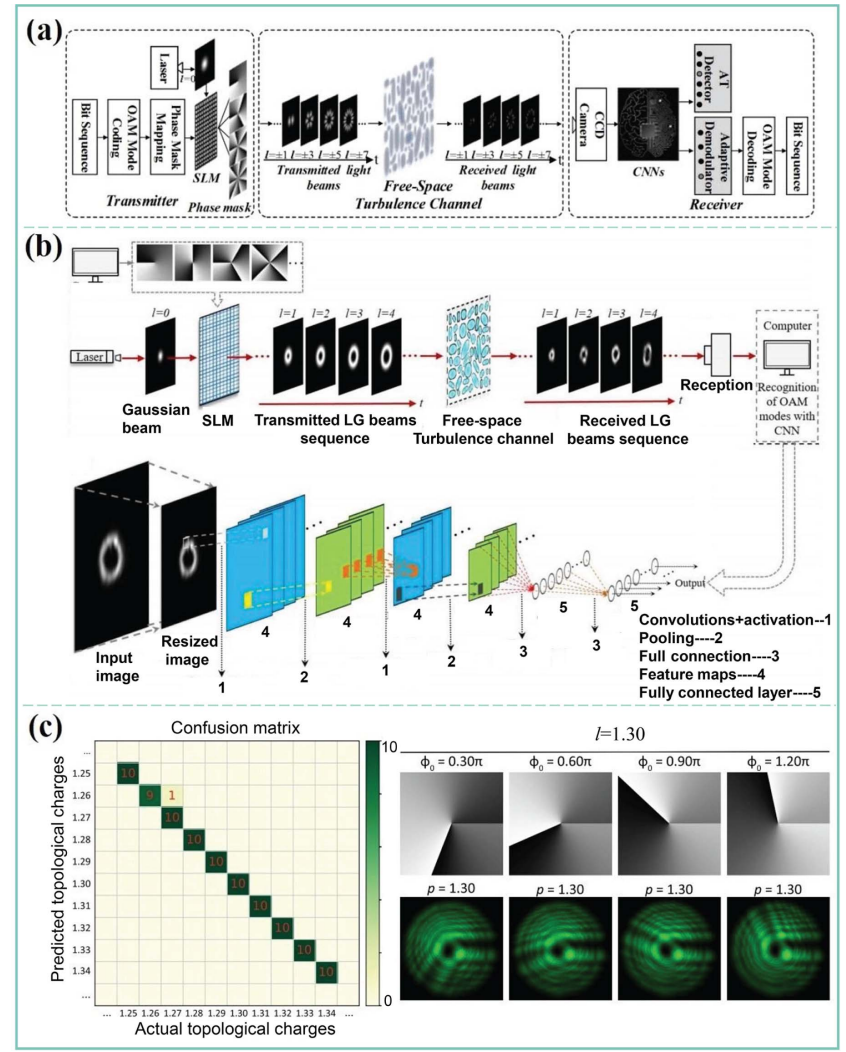

Fig. 9. (a) Numerical model of the OAM-based communication system. CCD camera, charge-coupled device camera ${ }^{[112]}$. (b) CNN-based OAM transmission system scheme. OAM transmission system block diagram, six-layer CNN $\operatorname{architecture}^{[113]}$. (c) Quantitative analysis of CNN and the recognized OAM modes with fractional topological charge. Confusion matrix from $I=1.25$ to $I=1.34$. First row: phase pictures uploaded on the SLM. Second row: intensity distributions of vortex modes recorded by the CMOS camera ${ }^{[115]}$.

LG beams to obtain better recognition performance and higher accuracy $^{[111]}$.

In addition, Liu et al. proposed a superhigh-resolution recognition scheme based on deep learning with fractional topological charges of the 0.01 level. Through changing the phase, the same OAM mode can get different intensity maps, which can generate a large number of intensity maps and send them to neural network training, as shown in Fig. 9(c) ${ }^{[115]}$. To detect high OAM numbers, Knutson et al. utilized deep neural networks to realize the recognition of an OAM value up to $100^{[116]}$. Different amounts of Gaussian white noise and multiplicative noise are added to the vortex beam images to simulate non-ideal experimental conditions. First, a large number of training images are produced and sent to the 16-layer neural network for learning. Moreover, Giordani et al. used polarizers to obtain the projection intensity of the vector vortex beam in various directions. Then, different Stokes parameters with RGB colors are calculated and coded to obtain a large number of pictures. Finally, the CNN network is used to identify the vector vortex beam ${ }^{[17]}$.

As a consequence, with the advantages of low cost, high speed, high precision, low device processing difficulty, and no 
redundant optical equipment, the OAM recognition scheme based on deep learning is still a research hotspot in the field of OAM optical communication. The technology has potential application value for realizing large-capacity optical communication networks based on vortex beams in the future.

\subsection{Using surface plasmon polaritons}

In conventional methods, regulating the light field by accumulating propagation distance makes it rather bulky, and it is difficult to break the diffraction limit. Owing to the excellent ability of manipulating the nanoscale field, surface plasmon polaritons have aroused interest to detect the OAM of vortex beams, such as on-chip plasmonic nanogratings ${ }^{[118-122]}$. In 2012, Capasso et al. found that the excited plasmonic wave will converge into a spot if a vortex beam is incident on a holographic grating with matched parameters, and thus its topological charge can be detected ${ }^{[118]}$. However, each plasmonic coupler can only measure a vortex beam with specific topological charge. Subsequently, a semi-ring plasmonic nanoslit was proposed for the discrimination of OAM modes, as shown in Fig. 10(a). After vortex beams are illuminated on the nanoslits, surface plasmon polaritons can be excited and focused into subwavelength spots at different locations related to topological charge $l$ owing to constructive interference, the interval of which is around $100 \mathrm{~nm}$ between adjacent states ${ }^{[119]}$. Based on this principle, OAM states of incident vortex beams can be detected through positions of multiple focal points. The limitation of such a device to the interval of neighboring OAM modes is relatively small. Also, the incident lights are restrained so that only circularly polarized vortex beams can be measured.

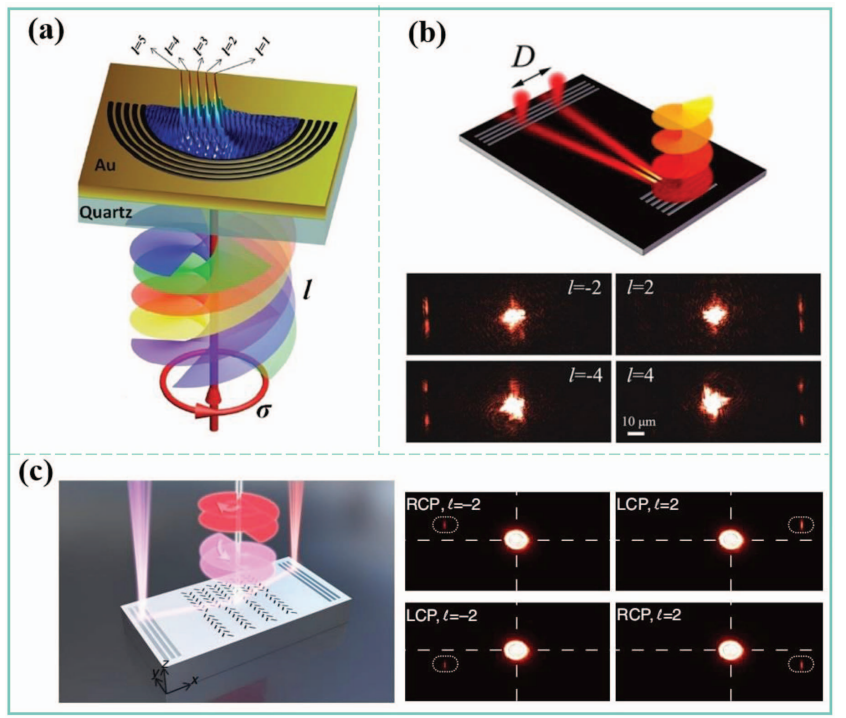

Fig. 10. Methods for detecting OAM modes based on surface plasmon polariton. (a) A semi-ring plasmonic nanoslit ${ }^{[119]}$. (b) A nanograting with straight stripes $^{[121]}$. (c) A plasmonic spin-Hall nanograting ${ }^{[122]}$.
Later, Chen et al. proposed another kind of plasmonic nanograting with fringes to couple OAM modes into two bifurcated surface plasmon polaritons with different splitting angles. As shown in Fig. 10(b), the splitting angles of plasmonic waves are strongly dependent on the topological charges of incident vortex beams, and thus OAM modes can be detected by different angles without particular alignment ${ }^{[121]}$. Also, a composite plasmonic nanograting was designed to detect the sign of topological charges, which can unidirectionally couple OAM modes into surface plasmon polaritons propagating on one side only. As such, a full measurement of OAM states can be implemented with the single device. The absolute value of the topological charge can be discriminated by the angle of launched plasmonic waves, the sign of which can be measured through its direction. The above on-chip nanogratings can only achieve the detection of OAM states rather than the SAM of incident lights. In 2020, a plasmonic spin-Hall nanograting was designed to measure both the polarization and phase singularities of incident cylindrical vortex vector beams ${ }^{[122]}$. As shown in Fig. 10(c), spin-Hall meta-slits are integrated onto the symmetry-breaking grating so that a chiral response can be observed for the discrimination of polarization. Such on-chip nanogratings would benefit diverse applications with great potential in integrated photonic circuits.

\section{Conclusion}

Vortex beams carrying OAM have broadened the perspectives in the optical realm owing to unique properties. In this review, we have summarized approaches and recent advances in the field of vortex beams generation and OAM detection. Classical optical elements such as SPP can generate vortex beams with extremely high conversion efficiency, whereas the requirement for manufacturing techniques is rather high. With the development of liquid crystal technology, digital devices are commonly used to produce vortex beams for their versatility. Moreover, binary amplitude masks such as photon and electron sieves can also be adopted for the generation of vortex beams. Nanofabrication technology makes it possible to generate such beams via ultrathin optical devices such as metasurfaces. As for the detection methods, the interferometer and diffraction grating can only detect a single topological charge at a time. In recent years, methods using geometric coordinate transformation, deep learning, and on-chip plasmonic nanogratings can further realize the recognition of complex OAM modes. We review the methods for generating and detecting the OAM in a concise way to facilitate further research on the vortex beams.

Notwithstanding the aforementioned approaches, there still exist challenges for both generation and detection of OAM. For example, the switch speed of SLMs usually hinders real-time applications in experiments. Promising advances can be expected once the technology becomes more mature. As for the detection methods, surface plasmon polaritons, for instance, have certain limitations such as strict alignment of incident lights, despite that such a method can promote the development 
of integrated photonic systems. Hopefully, vortex beams in light, as well as other forms of waves, will continue to thrive and enable new applications in many other fields ${ }^{[123,124]}$.

\section{Acknowledgement}

This work was supported by the National Natural Science Foundation of China (Nos. 11874102 and 12174047), Sichuan Province Science and Technology Support Program (No. 2020JDRC0006), and Fundamental Research Funds for the Central Universities (No. ZYGX2019J102).

\section{References}

1. J. H. Poynting, "The wave motion of a revolving shaft, and a suggestion as to the angular momentum of a beam of circularly polarised light," Proc. R. Soc. Lond. A 82, 560 (1909).

2. C. G. Darwin, "Notes on the theory of radiation," Proc. R. Soc. Lond. A 136, 36 (1932).

3. L. Allen, M. W. Beijersbergen, R. J. C. Spreeuw, and J. P. Woerdman, "Orbital angular momentum of light and the transformation of Laguerre-Gaussian laser modes,” Phys Rev. A 45, 8185 (1992).

4. A. Yao and M. J. Padgett, "Orbital angular momentum: origins, behavior and applications," Adv. Opt. Photon. 3, 161 (2011).

5. J. Durnin, J. J. Miceli, Jr., and J. Eberly, "Diffraction-free beams," Phys. Rev. Lett. 58, 1499 (1987).

6. J. Gutierrez-Vega, M. Iturbe-Castillo, and S. Chavez-Cerda, "Alternative formulation for invariant optical fields: Mathieu beams," Opt. Lett. 25, 1493 (2000).

7. N. Bozinovic, Y. Yue, Y. Ren, T. P. Kristensen, H. Huang, A. E. Willner, and S. Ramachandran, "Terabit-scale orbital angular momentum mode division multiplexing in fibers," Science 340, 1545 (2013).

8. H. He, M. E. Friese, N. R. Heckenberg, and H. Rubinsztein-Dunlop, "Direct observation of transfer of angular momentum to absorptive particles from a laser beam with a phase singularity," Phys. Rev. Lett. 75, 826 (1995).

9. J. Leach, B. Jack, J. Romero, A. K. Jha, A. Yao, S. Franke-Arnold, D. G. Ireland, R. W. Boyd, S. M. Barnett, and M. J. Padgett, "Quantum correlations in optical angle-orbital angular momentum variables," Science 329, 662 (2010).

10. P. Chen, L. Ma, W. Duan, J. Chen, S. Ge, Z. Zhu, M. Tang, R. Xu, W. Gao, T. Li, W. Hu, and Y. Lu, "Digitalizing self-assembled chiral superstructures for optical vortex processing," Adv. Mater. 30, 1705865 (2018).

11. P. Chen, L. Ma, W. Hu, Z. Shen, H. K. Bisoyi, S. Wu, S. Ge, Q. Li, and Y. Lu, "Chirality invertible superstructure mediated active planar optics," Nat. Commun. 10, 2518 (2019).

12. M. W. Beijersbergen, L. Allen, H. van der Veen, and J. P. Woerdman, "Astigmatic laser mode converters and transfer of orbital angular-momentum," Opt. Commun. 112, 321 (1993).

13. M. W. Beijersbergen, R. P. C. Coerwinkel, M. Kristensen, and J. P. Woerdman, "Helical-wavefront laser beams produced with a spiral phaseplate," Opt. Commun. 112, 321 (1994).

14. S. S. R. Oemrawsingh, J. A. W. Van Houwelingen, E. R. Eliel, J. P. Woerdman, E. J. K. Verstegen, and J. G. Kloosterboer, "Production and characterization of spiral phase plates for optical wavelengths," Appl. Opt. 43, 688 (2004).

15. W. Lee, X. Yuan, and W. Cheong, "Optical vortex beam shaping by use of highly efficient irregular spiral phase plates for optical micromanipulation," Opt. Lett. 29, 1796 (2004).

16. C. Rotschild, S. Zommer, S. Moed, O. Hershcovitz, and S. G. Lipson, "Adjustable spiral phase plate," Appl. Opt. 43, 2397 (2004).

17. W. Wu, Z. Sheng, and $\mathrm{H}$. Wu, "Design and application of flat spiral phase plate," Acta Phys. Sin. 68, 054102 (2019).

18. A. Vijayakumar, C. Rosales-Guzman, M. R. Rai, J. Rosen, O. V. Minin, I. V. Minin, and A. Forbes, "Generation of structured light by multilevel orbital angular momentum holograms," Opt. Express 27, 6459 (2019).
19. V. Bazhenov, M. Vasnetsov, and M. Soskin, "Laser beams with screw dislocations in their wavefronts," JETP Lett. 52, 429 (1990).

20. V. Bazhenov, M. S. Soskin, and M. V. Vasnetsov, "Screw dislocations in light wavefronts," J. Mod. Opt. 39, 985 (2007).

21. L. Stoyanov, S. Topuzoski, I. Stefanov, L. Janicijevic, and A. Dreischuh, "Far field diffraction of an optical vortex beam by a fork-shaped grating," Opt. Commun. 350, 301 (2015)

22. N. R. Heckenberg, R. McDuff, C. P. Smith, and A. G. White, "Generation of optical phase singularities by computer-generated holograms," Opt. Lett. 17, 221 (1992).

23. A. Sakdinawat and Y. Liu, "Soft-X-ray microscopy using spiral zone plates," Opt. Lett. 32, 2635 (2007).

24. K. Saitoh, Y. Hasegawa, N. Tanaka, and M. Uchida, "Production of electron vortex beams carrying large orbital angular momentum using spiral zone plates," J. Electron. Microsc. 61, 171 (2012).

25. C. Rosales-Guzmán and A. Forbes, How to Shape Light with Spatial Light Modulators (SPIE, 2017).

26. G. Gibson, J. C. Miles, and M. J. Padgett, "Free-space information transfer using light beams carrying orbital angular momentum," Opt. Express 12, 5448 (2004).

27. R. S. Nesbitt, S. L. Smith, R. A. Molnar, and S. A. Benton, "Holographic recording using a digital micromirror device," Proc. SPIE 3637, 12 (1999).

28. K. J. Mitchell, S. Turtaev, M. J. Padgett, T. Cizmar, and D. B. Phillips, "Highspeed spatial control of the intensity, phase and polarisation of vector beams using a digital micro-mirror device," Opt. Express 24, 29269 (2016).

29. W. H. Lee, "Binary synthetic holograms," Appl. Opt. 13, 1677 (1974).

30. V. Lerner, D. S. Drori, and N. Katz, "Shaping Laguerre-Gaussian laser modes with binary gratings using a digital micromirror device," Opt. Lett. 37, 4826 (2012).

31. Y. Ren, M. Huang, Z. Wang, J. Wu, and Y. Li, "Experimental generation of Laguerre-Gaussian beam using digital micromirror device," Appl. Opt. 49, 1838 (2010).

32. M. Mirhosseini, O. S. M. Loaiza, C. Chen, B. Rodenburg, M. Malik, and R. W. Boyd, "Rapid generation of light beams carrying orbital angular momentum," Opt. Express 21, 30196 (2013).

33. Y. Chen, Z. Fang, Y. Ren, L. Gong, and R. Lu, "Generation and characterization of a perfect vortex beam with a large topological charge through a digital micromirror device," Appl. Opt. 54, 8030 (2015).

34. S. A. Goorden, J. Bertolotti, and A. P. Mosk, "Superpixel-based spatial amplitude and phase modulation using a digital micromirror device," Opt. Express 22, 17999 (2014).

35. L. Kipp, M. Skibowski, R. L. Johnson, R. Berndt, R. Adelung, S. Harm, and R. Seemann, "Sharper images by focusing soft X-rays with photon sieves," Nature 414, 184 (2001).

36. K. Huang, H. Liu, F. J. Garcia-Vidal, M. Hong, B. Luk'yanchuk, J. Teng, and C. W. Qiu, "Ultrahigh-capacity non-periodic photon sieves operating in visible light," Nat. Commun. 6, 7059 (2015).

37. C. Xie, X. Shi, and M. Liu, "Spiral photon sieves apodized by digital prolate spheroidal window for the generation of hard-X-ray vortex," Opt. Lett. 35, 1765 (2010).

38. R. Liu, F. Li, M. J. Padgett, and D. B. Phillips, "Generalized photon sieves: fine control of complex fields with simple pinhole arrays," Optica 2, 1028 (2015).

39. Y. Yang, G. Thirunavukkarasu, M. Babiker, and J. Yuan, "Orbital-angularmomentum mode selection by rotationally symmetric superposition of chiral states with application to electron vortex beams," Phys. Rev. Lett. 119, 094802 (2017).

40. Y. Yang, Q. Zhao, L. Liu, Y. Liu, C. Rosales-Guzmán, and C. Qiu, "Manipulation of orbital-angular-momentum spectrum using pinhole plates," Phys. Rev. A 12, 064007 (2019).

41. J. Lin, P. Genevet, M. A. Kats, N. Antoniou, and F. Capasso, "Nanostructured holograms for broadband manipulation of vector beams," Nano Lett. 13, 4269 (2013).

42. C. Min, J. Liu, T. Lei, G. Si, Z. Xie, J. Lin, L. Du, and X. Yuan, "Plasmonic nano-slits assisted polarization selective detour phase meta-hologram," Laser Photon. Rev. 10, 978 (2016).

43. Z. Xie, T. Lei, G. Si, X. Wang, J. Lin, C. Min, and X. Yuan, "Meta-holograms with full parameter control of wavefront over a $1000 \mathrm{~nm}$ bandwidth," ACS Photon. 4, 2158 (2017). 
44. Z. Deng, J. Deng, X. Zhuang, S. Wang, T. Shi, G. Wang, Y. Wang, J. Xu, Y. Cao, X. Wang, X. Cheng, G. Li, and X. Li, "Facile metagrating holograms with broadband and extreme angle tolerance," Light-Sci. Appl. 7, 78 (2018).

45. Y. Zhang, W. Liu, J. Gao, and X. Yang, "Generating focused 3D perfect vortex beams by plasmonic metasurfaces," Adv. Opt. Mater. 6, 1701228 (2018).

46. F. Yue, D. Wen, and J. Xin, "Vector vortex beam generation with a single plasmonic metasurface," ACS Photon. 3, 1558 (2016).

47. R. Dharmavarapu, K. Izumi, I. Katayama, S. H. Ng, J. Vongsvivut, M. J. Tobin, A. Kuchmizhak, Y. Nishijima, S. Bhattacharya, and S. Juodkazis, "Dielectric cross-shaped resonator based metasurface for vortex beam generation in mid-IR and THz wavelengths," Nanophotonics 8, 1263 (2019).

48. C. Min, J. Liu, T. Lei, G. Si, Z. Xie, J. Lin, L. Du, and X. Yuan, "Plasmonic nano-slits assisted polarization selective detour phase meta-hologram," Laser Photon. Rev. 10, 978 (2016).

49. M. Khorasaninejad, W. Chen, R. C. Devlin, J. Oh, A. Zhu, and F. Capasso, "Metalenses at visible wavelengths: diffraction-limited focusing and subwavelength resolution imaging," Science 352, 1190 (2016).

50. M. L. N. Chen, L. Jiang, and W. E. I. Sha, "Ultrathin complementary metasurface for orbital angular momentum generation at microwave frequencies," IEEE Trans. Antenn. Propag. 65, 396 (2017)

51. A. Zhao, A. Pham, and A. Drezet, "Plasmonic fork-shaped hologram for vortex-beam generation and separation," Opt. Lett. 46, 689 (2021).

52. A. V. Kildishev, A. Boltasseva, and V. M. Shalaev, "Planar photonics with metasurfaces," Science 339, 1232009 (2013).

53. J. Sun, X. Wang, T. Xu, Z. A. Kudyshev, A. N. Cartwright, and N. M. Litchinitser, "Spinning light on the nanoscale," Nano. Lett. 14, 2726 (2014).

54. R. C. Devlin, A. Ambrosio, N. A. Rubin, J. P. B. Mueller, and F. Capasso, "Arbitrary spin-to-orbital angular momentum conversion of light," Science 358, 896 (2017).

55. M. Q. Mehmood, S. Mei, S. Hussain, K. Huang, S. Y. Siew, L. Zhang, T. Zhang, X. Ling, H. Liu, J. Teng, A. Danner, S. Zhang, and C. Qiu, "Visible-frequency metasurface for structuring and spatially multiplexing optical vortices," Adv. Mater. 28, 2533 (2016).

56. Y. Shi, B. Shen, L. Zhang, X. Zhang, W. Wang, and Z. Xu, "Light fan driven by a relativistic laser pulse," Phys. Rev. Lett. 112, 235001 (2014).

57. M. Beresna, M. Gecevicius, and P. G. Kazansky, "Polarization sensitive elements fabricated by femtosecond laser nanostructuring of glass," Opt. Mater. Express 1, 783 (2011).

58. M. Gecevicius, R. Drevinskas, M. Beresna, and P. G. Kazansky, "Single beam optical vortex tweezers with tunable orbital angular momentum," Appl. Phys. Lett. 104, 231110 (2014).

59. J. A. Davis, N. H. M. Kurihara, E. Hurtado, M. Pierce, M. M. Sanchez-Lopez, K. Badham, and I. Moreno, "Analysis of a segmented q-plate tunable retarder for the generation of first-order vector beams," Appl. Opt. 54, 9583 (2015).

60. L. Marrucci, C. Manzo, and D. Paparo, "Optical spin-to-orbital angular momentum conversion in inhomogeneous anisotropic media," Phys. Rev. Lett. 96, 163905 (2006)

61. J. F. Bisson, J. Li, K. Ueda, and Y. Senatsky, "Generation of LaguerreGaussian modes in Nd:YAG laser using diffractive optical pumping," Laser. Phys. Lett. 2, 327 (2005).

62. A. Ito, Y. Kozawa, and S. Sato, "Generation of hollow scalar and vector beams using a spot-defect mirror," J. Opt. Soc. Am. A 27, 2072 (2010).

63. M. Okida, T. Omatsu, M. Itoh, and T. Yatagai, "Direct generation of high power Laguerre-Gaussian output from a diode-pumped Nd:YVO $1.3 \mu \mathrm{m}$ bounce laser," Opt. Express 15, 7616 (2007).

64. G. Porat, I. Dolev, O. Barlev, and A. Arie, "Airy beam laser," Opt. Lett. 36, 4119 (2011).

65. Y. Yang, L. Wu, Y. Liu, D. Xie, Z. Jin, J. Li, G. Hu, and C. Qiu, "Deuterogenic plasmonic vortices," Nano. Lett. 20, 6774 (2020).

66. L. Wu, X. Li, and Y. Yang, "Generation of surface plasmon vortices based on double-layer Archimedes spirals," Acta Phys. Sin. 68, 234201 (2019).

67. T. W. Ebbesen, H. J. Lezec, H. F. Ghaemi, T. Thio, and P. A. Wolff, "Extraordinary optical transmission through sub-wavelength hole arrays," Nature 391, 667 (1998).

68. H. J. Lezec, A. Degiron, E. Devaux, R. A. Linke, L. Martin-Moreno, F. J. Garcia-Vidal, and T. W. Ebbesen, "Beaming light from a subwavelength aperture," Science 297, 820 (2002).
69. G. M. Lerman, A. Yanai, and U. Levy, "Demonstration of nanofocusing by the use of plasmonic lens illuminated with radially polarized light," Nano Lett. 9, 2139 (2009).

70. H. L. Offerhaus, B. van den Bergen, M. Escalante, F. B. Segerink, J. P. Korterik, and N. F. van Hulst, "Creating focused plasmons by noncollinear phasematching on functional gratings," Nano Lett. 5, 2144 (2005).

71. Y. Yang, Y. Ren, M. Chen, Y. Arita, and C. Rosales-Guzmán, "Optical trapping with structured light: a review," Adv. Photon. 3, 034001 (2021).

72. M. E. J. Friese, T. A. Nieminen, N. R. Heckenberg, and H. RubinszteinDunlop, "Optical alignment and spinning of laser-trapped microscopic particles," Nature 394, 348 (1998).

73. A. Mair, A. Vaziri, G. Weihs, and A. Zeilinger, "Entanglement of the orbital angular momentum states of photons," Nature 412, 313 (2001).

74. G. Molina-Terriza, J. P. Torres, and L. Torner, "Twisted photons," Nat. Phys. 3, 305 (2007).

75. J. Vickers, M. Burch, R. Vyas, and S. Singh, "Phase and interference properties of optical vortex beams," J. Opt. Soc. Am. A 25, 823 (2008).

76. H. Huang, Y. Ren, Y. Yan, N. Ahmed, Y. Yue, A. Bozovich, B. I. Erkmen, K. Birnbaum, S. Dolinar, M. Tur, and A. E. Willner, "Phase-shift interference-based wavefront characterization for orbital angular momentum modes," Opt. Lett. 38, 2348 (2013).

77. M. Harris, C. A. Hill, P. R. Tapster, and J. M. Vaughan, "Laser modes with helical wave fronts," Phys. Rev. A 49, 3119 (1994).

78. L. Chen, W. Zhang, Q. Lu, and X. Lin, "Making and identifying optical superpositions of high orbital angular momenta," Phys. Rev. A 88, 053831 (2013).

79. H. I. Sztul and R. R. Alfano, "Double-slit interference with Laguerre Gaussian beams," Opt. Lett. 31, 999 (2006).

80. O. Emile and J. Emile, "Young's double-slit interference pattern from a twisted beam," Optica 2, 1028 (2015).

81. T. Chen, X. Lu, J. Zeng, Z. Wang, H. Zhang, C. Zhao, B. J. Hoenders, and Y. Cai, "Young's double-slit experiment with a partially coherent vortex beam," Opt. Express 28, 38106 (2020).

82. W. Qi, R. Liu, and L. Kong, "Double-slit interference of single twisted photons," Chin. Opt. Lett. 18, 102601 (2020).

83. H. Zhou, L. Shi, X. Zhang, and J. Dong, "Dynamic interferometry measurement of orbital angular momentum of light," Opt. Lett. 39, 6058 (2014).

84. D. Fu, D. Chen, R. Liu, Y. Wang, H. Gao, F. Li, and P. Zhang, "Probing the topological charge of a vortex beam with dynamic angular double slits," Opt. Lett. 40, 788 (2015).

85. M. Malik, S. Murugkar, J. Leach, and R. W. Boyd, "Measurement of the orbital-angular-momentum spectrum of fields with partial angular coherence using double-angular-slit interference," Phys. Rev. A 86, 063806 (2012).

86. G. C. Gregorius and M. W. Beijersbergen, "Method for probing the orbital angular momentum of optical vortices in electromagnetic waves from astronomical objects," Phys. Rev. Lett. 101, 100801 (2008).

87. Q. Zhao, M. Dong, Y. Bai, and Y. Yang, "Measuring high orbital angular momentum of vortex beams with improved multipoint interferometer," Photon. Res. 8, 745 (2020).

88. J. Courtial, K. Dholakia, D. A. Robertson, L. Allen, and M. J. Padgett, "Measurement of the rotational frequency shift imparted to a rotating light beam possessing orbital angular momentum," Phys. Rev. Lett. 80, 3217 (1998).

89. J. Leach, M. J. Padgett, S. M. Barnett, S. Franke-Arnold, and J. Courtial, "Measuring the orbital angular momentum of a single photon," Phys. Rev. Lett. 88, 257901 (2002).

90. S. Slussarenko, V. D. Ambrosio, B. Piccirillo, L. Marrucci, and E. Santamato, "The polarizing sagnac interferometer: a tool for light orbital angular momentum sorting and spin-orbit photon processing," Opt. Express 18, 27205 (2010)

91. W. Zhang, Q. Qi, J. Zhou, and L. Chen, "Mimicking Faraday rotation to sort the orbital angular momentum of light," Phys. Rev. Lett. 112, 153601 (2014).

92. J. M. Hickmann, E. J. S. Fonseca, W. C. Soares, and S. Chavez-Cerda, "Unveiling a truncated optical lattice associated with a triangular aperture using light's orbital angular momentum," Phys. Rev. Lett. 105, 053904 (2010).

93. L. E. Araujo and M. E. Anderson, "Measuring vortex charge with a triangular aperture," Opt. Lett. 36, 787 (2011).

94. Y. Liu, H. Tao, J. Pu, and B. Lu, "Detecting the topological charge of vortex beams using an annular triangle aperture," Opt. Laser Technol. 43, 1233 (2011). 
95. J. G. Silva, A. J. Jesus-Silva, M. A. R. C. Alencar, J. M. Hickmann, and E. J. S. Fonseca, "Unveiling square and triangular optical lattices: a comparative study," Opt. Lett. 39, 949 (2014).

96. I. Moreno, J. A. Davis, B. M. L. Pascoguin, M. J. Mitry, and D. M. Cottrell, "Vortex sensing diffraction gratings," Opt. Lett. 34, 2927 (2009).

97. K. Dai, C. Gao, L. Zhong, Q. Na, and Q. Wang, "Measuring OAM states of light beams with gradually-changing-period gratings," Opt. Lett. 40, 562 (2015).

98. Y. Peng, X. Gan, P. Ju, Y. Wang, and J. Zhao, "Measuring topological charges of optical vortices with multi-singularity using a cylindrical lens," Chin. Phys. Lett. 32, 024201 (2015).

99. V. V. Kotlyar, A. A. Kovalev, and A. P. Porfirev, "Astigmatic transforms of an optical vortex for measurement of its topological charge," Appl. Opt. 56, 4095 (2017)

100. Z. Shuang and J. Wang, "Measuringorbital angular momentum (OAM) states of vortex beams with annular gratings," Sci. Rep. 7, 40781 (2017).

101. G. C. G. Berkhout, M. P. J. Lavery, J. Courtial, M. W. Beijersbergen, and M. J. Padgett, "Efficient sorting of orbital angular momentum states of light," Phys. Rev. Lett. 105, 153601 (2010).

102. G. C. G. Berkhout, M. P. J. Lavery, M. J. Padgett, and M. W. Beijersbergen, "Measuring orbital angular momentum superpositions of light by mode transformation," Opt. Lett. 36, 1863 (2011).

103. Y. Wen, I. Chremmos, Y. Chen, J. Zhu, Y. Zhang, and S. Yu, "Spiral transformation for high-resolution and efficient sorting of optical vortex modes," Phys. Rev. Lett. 120, 193904 (2018).

104. Y. Guo, S. Zhang, M. Pu, Q. He, J. Jin, M. Xu, Y. Zhang, P. Gao, and X. Luo, "Spin-decoupled metasurface for simultaneous detection of spin and orbital angular momenta via momentum transformation," Light-Sci. Appl. 10, 63 (2021).

105. G. Ruffato, M. Massari, and F. Romanato, "Compact sorting of optical vortices by means of diffractive transformation optics," Opt. Lett. 42, 551 (2017).

106. G. Ruffato, M. Massari, M. Girardi, G. Parisi, M. Zontini, and F. Romanato, "Non-paraxial design and fabrication of a compact OAM sorter in the telecom infrared," Opt. Express 27, 24123 (2019).

107. S. Lightman, G. Hurvitz, R. Gvishi, and A. Arie, "Miniature wide-spectrum mode sorter for vortex beams produced by 3D laser printing," Optica 4, 605 (2017).

108. Y. Fu, C. Min, J. Yu, Z. Xie, G. Si, X. Wang, Y. Zhang, T. Lei, J. Lin, D. Wang, H. P. Urbach, and X. Yuan, "Measuring phase and polarization singularities of light using spin-multiplexing metasurfaces," Nanoscale 11, 18303 (2019).

109. S. Zhang, P. Huo, W. Zhu, C. Zhang, P. Chen, M. Liu, L. Chen, H. J. Lezec, A. Agrawal, Y. Lu, and T. Xu, "Broadband detection of multiple spin and orbital angular momenta via dielectric metasurface," Laser Photon. Rev. 14, $2000062(2020)$
110. Y. LeCun, Y. Bengio, and G. Hinton, “Deep learning," Nature 521, 436 (2015).

111. M. I. Dedo, Z. Wang, K. Guo, and Z. Guo, "OAM mode recognition based on joint scheme of combining the Gerchberg-Saxton (GS) algorithm and convolutional neural network (CNN)," Opt. Commun. 456, 124696 (2020).

112. J. Li, M. Zhang, D. Wang, S. Wu, and Y. Zhan, "Joint atmospheric turbulence detection and adaptive demodulation technique using the $\mathrm{CNN}$ for the OAM-FSO communication," Opt. Express 26, 10494 (2018).

113. Z. Wang, M. I. Dedo, K. Guo, K. Zhou, F. Shen, Y. Sun, S. Liu, and Z. Guo, "Efficient recognition of the propagated orbital angular momentum modes in turbulences with the convolutional neural network," IEEE Photon. J. 11, 7903614 (2019)

114. X. Fu, Y. Bai, and Y. Yang, "Measuring OAM by the hybrid scheme of interference and convolutional neural network," Opt. Eng. 60, 064109 (2021).

115. Z. Liu, S. Yan, H. Liu, and X. Chen, "Superhigh-resolution recognition of optical vortex modes assisted by a deep-learning method," Phys. Rev. Lett. 123, 183902 (2019)

116. K. M. Iftekharuddin, A. A. S. Awwal, M. García Vázquez, A. Márquez, M. A. Matin, E. M. Knutson, S. Lohani, O. Danaci, S. D. Huver, and R. T. Glasser, "Deep learning as a tool to distinguish between high orbital angular momentum optical modes," Proc. SPIE 9970, 997013 (2016).

117. T. Giordani, A. Suprano, E. Polino, F. Acanfora, L. Innocenti, A. Ferraro, M. Paternostro, N. Spagnolo, and F. Sciarrino, "Machine learning-based classification of vector vortex beams," Phys. Rev. Lett. 124, 160401 (2020).

118. P. Genevet, J. Lin, M. A. Kats, and F. Capasso, "Holographic detection of the orbital angular momentum of light with plasmonic photodiodes," Nat. Commun. 3, 1278 (2012)

119. S. T. Mei, K. Huang, H. Liu, F. Qin, M. Q. Mehmood, Z. Xu, M. Hong, D. Zhang, J. Teng, A. Dannera, and C. Qiu, "On-chip discrimination of orbital angular momentum of light with plasmonic nanoslits," Nanoscale 8, 2227 (2016).

120. J. Chen, T. Li, S. Wang, and S. Zhu, "Multiplexed holograms by surface plasmon propagation and polarized scattering," Nano Lett. 17, 5051 (2017).

121. J. Chen, X. Chen, T. Li, and S. Zhu, "On-chip detection of orbital angular momentum beam by plasmonic nanogratings," Laser Photon. Rev. 12, 1700331 (2018)

122. F. Feng, G. Si, C. Min, X. Yuan, and M. Somekh, "On-chip plasmonic spinHall nanograting for simultaneously detecting phase and polarization singularities," Light-Sci. Appl. 9, 95 (2020).

123. M. Dong, C. Zhao, Y. Cai, and Y. Yang, "Partially coherent vortex beams: fundamentals and applications," Sci. China Phys. Mech. 64, 224201 (2021).

124. D. M. Fatkhiev, M. A. Butt, E. P. Grakhova, R. V. Kutluyarov, I. V. Stepanov, N. L. Kazanskiy, S. N. Khonina, V. S. Lyubopytov, and A. K. Sultanov, "Recent advances in generation and detection of orbital angular momentum optical beams: a review," Sensors 21, 4988 (2021). 